\title{
Processes of auroral thermal structure at Jupiter: Analysis of multispectral temperature observations with the Jupiter Thermosphere General Circulation Model
}

\author{
T. Majeed, ${ }^{1,2}$ J. H. Waite, ${ }^{3}$ S. W. Bougher, ${ }^{1}$ and G. R. Gladstone ${ }^{3}$ \\ Received 13 May 2008; revised 23 April 2009; accepted 6 May 2009; published 25 July 2009.
}

[1] We use our three-dimensional Jupiter Thermosphere General Circulation Model (JTGCM) to quantify thermal processes that take place in the auroral thermosphere. These processes tend to control the thermal budget in the Jovian ovals and polar caps and maintain thermospheric temperatures consistent with those derived from multispectral observations of Jupiter's aurora. The main heat source in the JTGCM that drives the thermospheric flow is high-latitude joule heating resulting from frictional motion of the ions relative to the neutrals. A secondary source of heating that dominates the exospheric region of the Jovian ovals is the auroral process of particle precipitation. Both sources of high-latitude heating in the JTGCM are strongly related to the current system in the outer magnetosphere that allows plasma to flow in and out of the Jovian ionosphere. The mapping of this flow to ionospheric altitudes gives rise to an ion drag process that dominates the neutral momentum forcing near the altitude of the ionospheric peak. We find that the ion drag and joule heating inputs in the JTGCM significantly intensify the underlying global thermospheric circulation, thereby affecting the distribution of the neutral temperature. Global simulations of the Jovian thermospheric dynamics indicate strong neutral outflows from the auroral ovals with velocities up to $1.9 \mathrm{~km} \mathrm{~s}^{-1}$ and subsequent convergence and downwelling at the Jovian equator. Such circulation is shown to be an important mechanism for transporting significant amounts of auroral energy to the rest of the planet and for regulating the global heat budget in a manner consistent with temperature observations of Jupiter's oval and polar cap regions. Adiabatic expansion of the neutral atmosphere resulting from outward flows is found to be an important source of cooling for the auroral exosphere. The distribution of neutral temperature from $1 \mu \mathrm{bar}$ to 1 nbar is determined by the thermal balance between the total heating caused by joule and adiabatic heating processes and dynamical cooling mainly from the hydrodynamic advection process. The thermal balance of the Jovian thermosphere below the homopause ( $\geq 1 \mu \mathrm{bar}$ ) is shown to be dominated primarily by wind transport processes. The heating in this region both for the Jovian ovals and polar caps is due to hydrodynamic advection and adiabatic compression processes. This dynamical heating is efficiently dissipated by hydrocarbon cooling through $\mathrm{CH}_{4}$ and $\mathrm{C}_{2} \mathrm{H}_{2}$ infrared radiation at 7.8 and $12.6 \mu \mathrm{m}$, respectively.

Citation: Majeed, T., J. H. Waite, S. W. Bougher, and G. R. Gladstone (2009), Processes of auroral thermal structure at Jupiter: Analysis of multispectral temperature observations with the Jupiter Thermosphere General Circulation Model, J. Geophys. Res., 114, E07005, doi:10.1029/2008JE003194.

\section{Introduction}

[2] Remote sensing of Jupiter at X-ray to radio wavelengths has revealed much about the characteristics of the Jovian aurora [Satoh et al., 1996; Prangé et al., 1998;

\footnotetext{
${ }^{1}$ Department of Atmospheric, Oceanic and Space Sciences, University of Michigan, Ann Arbor, Michigan, USA.

${ }^{2}$ Department of Physics, American University of Sharjah, Sharjah, United Arab Emirates.

${ }^{3}$ Southwest Research Institute, San Antonio, Texas, USA.

Copyright 2009 by the American Geophysical Union. 0148-0227/09/2008JE003194
}

Waite et al., 2000; Pallier and Prangé, 2001; Waite and Lummerzheim, 2002; Gustin et al., 2004a, 2004b, 2006; Elsner et al., 2005; Bhardwaj et al., 2007; Grodent et al., 2008; Branduardi-Raymont et al., 2008] and about the potential impact of the ionosphere-magnetosphere coupling that presumably drives the thermal and dynamical balances of Jupiter's upper atmosphere [Achilleos et al., 1998; Bunce and Cowley, 2001; Stallard et al., 2001; Cowley and Bunce, 2001; Cowley et al., 2003a, 2003b, 2005; Nichols and Cowley, 2004; Millward et al., 2005; Bougher et al., 2005]. Most importantly, analyses of all observations of Jupiter's aurora from the Voyager spacecraft in 1979 
[Broadfoot et al., 1979] to the present, including flybys and Earth orbiting telescope observations from ultraviolet to radio wavelengths [e.g., Elsner et al., 2005; Ajello et al., 2005; Gustin et al., 2006; Radioti et al., 2008; Grodent et al., 2008], suggest that Jupiter has a total auroral particle input energy flux of the order of $10^{13}-10^{14} \mathrm{~W}$ in each hemisphere. This is about 3 orders of magnitude stronger than the corresponding value for Earth's aurora [Grodent et al., 2001]. Since this energy is deposited over a relatively small region, the aurora constitutes a great perturbation on the Jovian upper atmosphere, perhaps even resulting in supersonic winds and a very hot thermosphere [Sommeria et al., 1995; Achilleos et al., 1998; Stallard et al., 2002; Bougher et al., 2005; Melin et al., 2006]. Our initial picture of the Jovian aurora, as primarily associated with the Io Plasma Torus (IPT), changed as high spatial resolution Hubble Space Telescope (HST) images became available [Caldwell et al., 1992; Dols et al., 2000; Gérard et al., 1994; Clarke et al., 1998; Ballester et al., 1996; Grodent et al., 2003; Radioti et al., 2008]. Supported by high-resolution ground-based imaging of $\mathrm{H}_{3}^{+}$emissions [e.g., Connerney et al., 1993], the early HST data clearly showed that Jupiter's main auroral oval originates much further out in the magnetosphere, by indicating that main oval emissions occur on the planet at much higher magnetic latitudes than where the IPT footprint falls. The main auroral oval emissions as observed in the UV by HST and other UV telescopes [see Grodent et al., 2008 , and references therein] are confined to a narrow latitude band mapping to a radial distance in the magnetosphere of about 20-30 $R_{j}$. Observations of the main oval emission also indicate that they corotate with the planet and have temporal variations on timescales from tens of minutes to hours [e.g., Grodent et al., 2008; Radioti et al., 2008]. The current theoretical explanation and mechanism of forming the main Jovian auroral oval is discussed in detail in section 2 .

[3] Recent analyses of far ultraviolet (FUV) [Grodent et al., 2003; Gérard et al., 2003; Gustin et al., 2004a, 2004b, 2006] and X-ray [Waite et al., 2001; Gladstone et al., 2002; Cravens et al., 2003; Branduardi-Raymont et al., 2007, 2008] images have also revealed intense and localized transient emissions poleward of the main oval that are magnetically connected to the outermost regions of Jupiter's magnetosphere (possibly $>30 R j$ ) consistent with the modeling results of Cowley et al. [2005]. These flare-like emissions may be an expression of the return (downward) current that closes the corotation breakdown circuit, as suggested by Cravens et al. [2003]. This conclusion recently gained support from Kharchenko et al. [2006] and Branduardi-Raymont et al. [2008]. Clearly, FUV auroral and $\mathrm{X}$-ray emissions represent the observable signature of largescale magnetospheric current systems, and the input power of the aurora is large enough to dominate the global energy budget of Jupiter's upper atmosphere and generate very strong neutral winds [Millward et al., 2005; Bougher et al., 2005; Majeed et al., 2005]. Furthermore, the near-infrared (near-IR) imaging (mostly in the $2-4 \mu \mathrm{m}$ range) of Jupiter's polar regions has been used to address auroral morphology and variability of $\mathrm{H}_{3}^{+}$emissions [Baron et al., 1996; Kim et al., 1991; Drossart et al., 1993; Satoh and Connerney, 1999], thereby opening up the possibility of remote sensing studies of the Jovian polar ionospheric flows. Several studies in this regard have now recognized $\mathrm{H}_{3}^{+}$as an important tracer of the thermospheric energetics [see Raynaud et al., 2004b; Melin et al., 2006, and references therein] and dynamics [Stallard et al., 2001; Rego et al., 1999; Maillard et al., 1999] owing to its coupling with Jupiter's outer magnetosphere [e.g., Cowley et al., 2005, and references therein]. Clearly, progress in imaging and spectroscopy of the Jovian aurora, over a wide range of wavelengths from IR to $\mathrm{X}$-ray, has greatly improved our knowledge of the thermospheric response induced by precipitating charged particles and has allowed us to reasonably estimate their impact on the thermal and dynamical structure of Jupiter's upper atmosphere [Bougher et al., 2005; Millward et al., 2005].

[4] While our views of Jupiter's auroral morphology have been evolving, much has also been learned through UV spectroscopy of the $\mathrm{H}_{2}$ Lyman and Werner band emissions. The Lyman and Werner bands are produced by direct electron excitation of the ground electronic state of $\mathrm{H}_{2}$, with some cascade contribution to the Lyman bands from electron excited higher-lying Rydberg singlet states. Spectral analysis of $\mathrm{H}_{2}$ band emissions provides useful constraints on the atmospheric temperature profile (through fitting of rovibrational bands) and the composition of the atmosphere (through absorption of the $\mathrm{H}_{2}$ emissions by overlying hydrocarbons, primarily $\mathrm{CH}_{4}$ and $\mathrm{C}_{2} \mathrm{H}_{2}$ ). Analyses by different groups of FUV and IR emission spectra have yielded effective $\mathrm{H}_{2}$ and $\mathrm{H}_{3}^{+}$temperatures and compositions representing the auroral regions. The derived temperatures, depending on the source of the data, are scattered over a wide range, as discussed in section 3. While some analyses obtained higher temperatures for the oval region than the polar region of the flares, given the huge energy input from the auroral particles and joule heating [e.g., Achilleos et al., 1998; Bougher et al., 2005; Millward et al., 2005]. One reason for the less than expected temperature rise is that cooling by $\mathrm{H}_{3}^{+}, \mathrm{CH}_{4}$, and $\mathrm{C}_{2} \mathrm{H}_{2}$ is so efficient. Another likely reason is that much of the input energy is converted to dynamical energy - neutral winds.

[5] The main objective of this paper is to interpret the derived temperatures from multispectral observations of Jupiter's auroral regions based on thermal balances calculated by our 3-D Jupiter Thermospheric General Circulation Model (JTGCM). The JTGCM has been used to simulate the global distribution of thermospheric winds, temperatures, and ion neutral species [e.g., Bougher et al., 2005; Majeed et al., 2005]. In this paper we use the JTGCM to characterize the relative roles of ion drag and joule heating in driving neutral thermospheric winds and the associated mapping of these drivers into the Jovian magnetosphere. We also demonstrate how the strong neutral wind system within the JTGCM is capable of regulating the bulk of the heating (with comparable cooling) in the thermosphere of the Jovian main oval emission region and polar cap region. Finally, the simulated vertical profiles of heating and cooling rates are used to quantify the processes responsible for maintaining the derived thermospheric temperatures.

\section{Recent Advances in Magnetospheric- Ionospheric Coupling and Dynamical Models}

[6] Modeling of dynamical magnetosphere-ionosphere coupling [e.g., Hill, 2001; Bunce and Cowley, 2001; Cowley 
and Bunce, 2001; Cowley et al., 2003a, 2003b, 2005; Nichols and Cowley, 2004; Millward et al., 2005] has shown that the supply of angular momentum to magnetospheric pickup ions originating from Io comes from the spinning down of Jupiter's atmosphere. The torque supplied through neutral atmosphere collisions with ionospheric particles results in near corotation of the ionosphere. The corotational electric field is subsequently transferred via field-aligned currents to the magnetodisk plasma that extends beyond the orbit of Io into the outer magnetosphere and onto open field lines [Mauk et al., 2002; Cowley et al., $2003 \mathrm{~b}]$. Corotation breakdown in the magnetodisk occurs when the distance along the magnetic field line and the associated finite angular momentum transfer time determined by the Alfvén speed of the medium is not sufficiently rapid to supply adequate angular momentum to a plasma parcel as it moves radially outward in the magnetodisk [e.g., Vasyliunas, 2001]. Timescales of Alfvénic communication along the field lines are of order minutes in the relevant region, while radial transport times are of order days, as estimated by Cowley and Bunce [2003, and references therein]. Thus, the magnetosphere and ionosphere remain in good communication along the field lines throughout the coupling process.

[7] The region of maximum change in the departure of the magnetodisk from corotation, or correspondingly the region of maximum radial shear in the magnetodisk, is in fact the region of maximum upward field-aligned (Birkeland) current, and thus is connected to the magnetic field lines that produce the main auroral oval [Hill, 2001; Cowley and Bunce, 2001; Khurana, 2001; Cowley et al., 2003a]. The electrons accelerated downward to auroral energies in the range 10-150 keV [Grodent et al., 2001; Ajello et al., 2001; Mauk et al., 2002; Gustin et al., 2004a, 2004b, 2006] in this process provide the field-aligned currents associated with corotation breakdown, and they produce UV emissions from electron excitation, as well as ionization and heating of the $\mathrm{H}_{2}$ gas. The heating due to this particle precipitation is a major driver of the thermospheric dynamics, since globally integrated solar heating of the thermosphere is approximately 100 times smaller than the globally integrated auroral heating [e.g., Strobel and Smith, 1973]. The strong horizontal closure currents near the auroral oval also produce joule heating in the ionosphere from ion neutral collisions between the ionosphere and the atmosphere, which, according to recent work [Nichols and Cowley, 2004; Millward et al., 2005; Bougher et al., 2005] supply the bulk of the heating in the Jovian upper atmosphere. The high-latitude joule and particle precipitation heating and subsequent equatorward winds produced in the thermosphere result in large jets via the Coriolis force [Achilleos et al., 1998; Bougher et al., 2005]. Because of the differential hemispheric response to the Coriolis force, the zonal jets in the southern hemisphere are in the opposite direction to the nearly corotating ionosphere, thus providing a quadratic feedback in the joule heating. In the northern hemisphere the opposite is true, and the joule heating is lessened. This produces a global asymmetry in the thermospheric wind system that blows light tracer constituents (e.g., atomic hydrogen atoms) to a preferential position in the northern hemisphere (see Bougher et al. [2005] for details).
[8] With regard to dynamical general circulation models of Jupiter, Achilleos et al. [1998] developed the first 3-D coupled ionosphere-thermosphere model called the Jovian Ionospheric Model (JIM). They used a monochromatic beam of auroral electrons characterized by an initial energy of $10 \mathrm{keV}$ and associated electron flux of $8 \mathrm{ergs} \mathrm{cm}^{-2} \mathrm{~s}^{-1}$ to demonstrate the role of the thermospheric wind system in controlling the global ionization and thermospheric heat budget. In a subsequent paper, Millward et al. [2002] summarized the JIM model and key simulations to investigate how ionospheric densities and conductivities respond to variations in electron precipitation within the auroral ovals. The goal was to study the impact of varying fluxes of auroral electrons ( 0.1 to $\left.1000 \mathrm{ergs} \mathrm{cm}^{-2} \mathrm{~s}^{-1}\right)$ and electron energies (1 to $1000 \mathrm{keV})$ on the neutral and ion structure. They also demonstrated the effect of auroral precipitation on the ionospheric conductivity and found that a beam of $60 \mathrm{keV}$ electrons associated with an upward field-aligned current of about $1 \mu \mathrm{Am}^{-2}$ can produce efficient auroral ionization, which leads to a maximum value of heightintegrated Pedersen conductivity of 7.5 mhos. In a subsequent paper, using similar auroral inputs to JIM coupled with equatorward transauroral voltage $(1-10 \mathrm{MV})$, Millward et al. [2005] investigated the dynamics of the coupling between the auroral ionosphere and the coexisting neutral thermosphere. They showed that ions subject to drift motion with velocities $>1 \mathrm{~km} \mathrm{~s}^{-1}$ around the auroral oval, as a result of the $E \times B$ coupling between a meridional, equatorward electric field and the Jovian magnetic field, generates neutral winds at thermospheric heights with velocities of $\sim 1 \mathrm{~km} \mathrm{~s}^{-1}$. They used the ratio of the difference between the angular velocities of Jupiter and the neutral gas in the Pedersen conductivity layer to the difference between the angular velocities of Jupiter and the plasma on the shell of field lines that magnetically connects from the magnetodisk to the ionospheric footprints to calculate the heightdependent ion neutral coupling coefficient, K. Millward et al. [2005] have also investigated the response of the Jovian ionosphere and Pedersen conductivity to varying incoming auroral electron number flux for a constant electron energy of $30 \mathrm{keV}$ and an applied auroral voltage of $3 \mathrm{MV}$ (equivalent to an electric field of $0.6 \mathrm{Vm}^{-1}$ ). They showed that the peak in the electron density profiles (at around $0.4 \mu \mathrm{bar}$ ) increases from $2 \times 10^{11} \mathrm{~m}^{-3}$ to $4 \times 10^{13} \mathrm{~m}^{-3}$ for a corresponding range of electron number flux of $2 \times 10^{11} \mathrm{~m}^{-2} \mathrm{~s}^{-1}$ to $2 \times 10^{14} \mathrm{~m}^{-2} \mathrm{~s}^{-1}$. The height-integrated Pedersen conductivity has also been shown to monotonically increase from 0.75 to 10 mhos for the above range of electron number flux.

[9] The neutral wind patterns at and near the auroral ionospheric peak may have a pronounced effect on the ion momentum forcing and the joule heating [Bougher et al., 2005]. Another consequence of the effects of dynamical feedback on the coupling processes is the effect on the electrical conductivity, which in turn affects the strength of the field-aligned currents [Nichols and Cowley, 2004]. Huang and Hill [1989] have shown that if the despinning of the atmosphere occurs because of the lack of vertical propagation of angular momentum, the height-integrated Pedersen conductivity $\left(\Sigma_{\mathrm{p}}\right)$ will decrease by a factor of $1-\mathrm{K}$. The reduced conductivity from its true value is referred 
to as the effective Pedersen conductivity $\left(\Sigma_{\mathrm{p}}^{*}\right)$. Thus the original value of $\mathrm{K}$ [e.g., Cowley and Bunce, 2001] was defined to represent this slippage of the ionosphere with respect to the corotational flow. However, in reality what occurs in the ion neutral coupling process is much more complicated. This has recently been investigated by Cowley et al. [2005] who modeled the plasma flow and currents in the polar ionosphere by employing both the internal magnetospheric plasma processes and the solar wind interaction. While the plasma angular velocities in the model were justified by using observed information on the plasma corotation in the inner $(\sim 10 R j)$, middle $(\sim 45 R j)$, and outer $(\sim 70 R j)$ magnetospheres, the emphasis was on the closure of upward field-aligned currents in different magnetospheric regions self-consistently with processes responsible for auroral precipitation. Since the flow of current in the magnetosphere-ionosphere system determines the strength of auroral precipitation, Cowley et al. [2005] discussed quantitative effects of constant and variable effective height-integrated Pedersen conductivity on an overall fieldaligned current structure within the middle magnetosphere, yielding precipitating accelerated electron energies and corresponding energy fluxes that reasonably explain main oval emissions observed in UV wavelengths. They have also shown that less intense UV emissions observed poleward of the main oval can be linked to reduced energy fluxes and to the total power of precipitating accelerated outer magnetospheric electrons as a result of spreading the upward fieldaligned current on closed field lines at the open field boundary. Interestingly, for the model with variable conductivity, Cowley et al. [2005] showed that $\Sigma_{\mathrm{p}}^{*}$ increases from a background value of 0.2 mhos to 1.5 mhos for field-aligned currents up to $0.6 \mu \mathrm{A} \mathrm{m}^{-2}$ flowing in the middle magnetosphere, and that in the outer magnetosphere the peak value of $\Sigma_{\mathrm{p}}^{*}$ is reduced to $\sim 0.7$ mhos for the source of fieldaligned currents. Although the study of the effects of upward field-aligned currents on the auroral precipitation process lies beyond the scope of this paper and is the subject of ongoing research, we will briefly discuss this later with our estimates of Pedersen conductivity obtained in this paper.

\section{High-Latitude Temperature Structure}

[10] The upper atmospheric structure of the Jovian auroral region has never been probed by in situ instrumentation. Instead, all our information on the thermal structure and composition has been derived from spectrographic and imaging data acquired with spacecraft and from groundbased and Earth-orbiting telescopes. There are the following three major sources for such information covering high altitudes: (1) thermal IR sounding of hydrocarbon emissions at pressures between $10^{-4}$ and $10^{-6}$ bars, (2) $\mathrm{H}_{2}$ rovibrational temperatures derived from UV auroral spectra from pressures in the range $10^{-5}-10^{-8}$ bars, and (3) rotational/vibrational temperatures derived from $\mathrm{H}_{3}^{+}$near-IR spectra emitted at pressures in the range $10^{-6}-10^{-10}$ bars. In section 3.1 , we review the details of temperatures derived from these various sources.

\subsection{Temperature Structure From Infrared Emission}

[11] Since its detection in a Jovian aurora in 1988 [e.g., Drossart et al., 1989], $\mathrm{H}_{3}^{+}$emissions have been used to probe auroral activity and morphology using both spectroscopic and imaging techniques [Miller et al., 1994]. Spectral mappings of $\mathrm{H}_{3}^{+}$emissions from the Jovian upper atmosphere have been obtained by Miller and coworkers [see Miller et al., 1997; Lam et al., 1997] using the CGS4 spectrometer on the United Kingdom Infrared Telescope (UKIRT), at Mauna Kea, Hawaii. These observations have been used to derive information on the upper atmospheric temperatures and $\mathrm{H}_{3}^{+}$column densities. An average temperature of $915 \pm 30 \mathrm{~K}$ was obtained for the southern auroral region while a relatively lower temperature of $734 \pm 37 \mathrm{~K}$ was obtained for the northern auroral region. The corresponding $\mathrm{H}_{3}^{+}$column densities were in the range $1-7 \times 10^{12} \mathrm{~cm}^{-2}$ (equivalent to an average pressure level close to $0.1 \mu \mathrm{bar}$ ).

[12] Additional information on temperatures has also been obtained from telescope facilities at four locations in North and South America during the occultation of the bright star HIP9369 by the northern polar regions of Jupiter. Raynaud et al. [2003] demonstrated that eight occultation profiles at different latitudes ranging from $55^{\circ} \mathrm{N}$ to $73.2^{\circ} \mathrm{N}$ show small-scale fluctuations in the inverted temperature profiles, perhaps due to propagating gravity waves [Young et al., 2005]. The derived temperatures from 50 to $2 \mu \mathrm{bar}$ were in the range 150-290 K. Raynaud et al. [2003] claimed that the variations in the retrieved temperature profiles were consistent with those derived from typical $\mathrm{CH}_{4}$ observations at $7.8 \mu \mathrm{m}$ [e.g., Orton et al., 1991]. Furthermore, a reanalysis of the 1971 Beta Scorpii occultation by the southern polar region of Jupiter allowed Raynaud et al. [2004a] to derive a stratospheric temperature profile ranging from 200 to $260 \mathrm{~K}$, consistent with that obtained by Veverka et al. [1974].

[13] Raynaud et al. [2004b] reported information on $\mathrm{H}_{3}^{+}$ temperature and composition, using spectroimaging IR observations of Jupiter's aurora obtained with the Fourier transform spectrometer (FTS)/Bear instrument at the CanadaFrance-Hawaii Telescope (CFHT). These observations were made near $2 \mu \mathrm{m}$, at moderately high spectral resolution $\left(0.2 \mathrm{~cm}^{-1}\right)$, and allowed them to map emissions from the $\mathrm{H}_{2} S_{1}$ (1) quadrupole line and from several $\mathrm{H}_{3}^{+}$lines of the $2 \nu_{2}$ overtone band. An interesting result from these observations was that the $\mathrm{H}_{3}^{+}$emission coincided in position with the "hot spot" region of increased and variable hydrocarbon, FUV, and X-ray emissions, while the $\mathrm{H}_{2} S_{1}$ (1) emission appeared to be more uniform. Despite their being observed simultaneously, the $\mathrm{H}_{2}$ and $\mathrm{H}_{3}^{+}$emission spectra appeared to be morphologically quite different. No viable explanation has yet been established for this scenario. The detection of several bright lines of $\mathrm{H}_{3}^{+}$(with different energy levels) allowed Raynaud et al. [2004b] to map $\mathrm{H}_{3}^{+}$temperatures and corresponding column densities in the Jovian auroral region, using the approach adopted by Stallard et al. [2002]. An analysis of a total of fourteen separate observations of both north and south auroral regions showed that the average distribution of $\mathrm{H}_{3}^{+}$temperature and composition is quite variable. An average temperature of $950 \pm 150 \mathrm{~K}$ in the north was obtained, with a corresponding $\mathrm{H}_{3}^{+}$column density of $(4 \pm 1) \times 10^{10} \mathrm{~cm}^{-2}$. A considerably warmer $\mathrm{H}_{3}^{+}$ temperature of $1275 \pm 175 \mathrm{~K}$ was derived for the south, with almost the same $\mathrm{H}_{3}^{+}$column density. The large difference in temperatures likely reflects the crucial role that the ionosphere-magnetosphere coupling plays in the energetics of the auroral regions, delivering substantial amounts of 
Table 1. Summary of Multispectral Observations of Jupiter's Temperatures ${ }^{\text {a }}$

\begin{tabular}{|c|c|c|c|}
\hline Experiment & Pressure (bar) & Effective Temperature (K) & Reference \\
\hline CFHT-FTS/Bear F2.12 filter (north) & $\sim 3 \times 10^{-10}$ & $925 \pm 70$ & Raynaud et al. [2004b] \\
\hline CFHT-FTS/Bear F2.09 filter (north) & $\sim 3 \times 10^{-10}$ & $1065 \pm 160$ & Raynaud et al. $[2004 \mathrm{~b}]$ \\
\hline CFHT-FTS/Bear F2.12 filter (south) & $\sim 3 \times 10^{-10}$ & $1275 \pm 160$ & Raynaud et al. [2004b] \\
\hline IRFT/Cshell & $\sim 1 \times 10^{-8}$ & $1000 \pm 50$ & Stallard et al. $[2001,2002]$ \\
\hline UV-FUSE $\mathrm{SiC}_{2} \mathrm{a}(920-970 \AA)$ & $1 \pm 0.3 \times 10^{-8}$ & $850 \pm 90$ & Gustin et al. $[2004 \mathrm{a}]$ \\
\hline UKIRT (north) & $\sim 7 \times 10^{-8}$ & $636 \pm 25$ & Miller et al. $[1997]$ \\
\hline UKIRT (south) & $\sim 8 \times 10^{-8}$ & $915 \pm 30$ & Miller et al. [1997] \\
\hline HST-STIS (north, main auroral oval) & $\sim 5 \times 10^{-7}$ & $475 \pm 65$ & Gustin et al. [2002] \\
\hline UV-FUSE LiF2a $(1090-1180 \AA)$ & $1.04 \pm 0.63 \times 10^{-6}$ & $800 \pm 45$ & Gustin et al. [2004a] \\
\hline UV-FUSE LiF1a (1030-1080 ̊) & $1.2 \pm 0.47 \times 10^{-6}$ & $800 \pm 90$ & Gustin et al. [2004a] \\
\hline GHRS/HST & $\sim 3 \times 10^{-6}$ & $500 \pm 40$ & Liu and Dalgarno [1996] \\
\hline HST-STIS (north, polar cap) & $\sim 3 \times 10^{-6}$ & $310 \pm 10$ & Gustin et al. [2002] \\
\hline HIP9369 occultation (north) & $\sim 5 \times 10^{-5}-1 \times 10^{-7}$ & $200-300$ & Raynaud et al. [2003] \\
\hline Beta Scorpii occultation (south) & $\sim 2 \times 10^{-4}-1 \times 10^{-6}$ & $200-230$ & Raynaud et al. [2004a] \\
\hline
\end{tabular}

${ }^{a}$ Here CFHT-FTS, Canada-France-Hawaii Telescope-Fourier transform spectrometer; IRFT, NASA Infrared Telescope Facility at Mauna Kea, Hawaii; FUSE, Far Ultraviolet Spectroscopic Explorer; UKIRT, United Kingdom Infrared Telescope; HST-STIS, Hubble Space Telescope-Space Telescope Imaging Spectrograph; GHRS, Goddard High-Resolution Spectrograph; HST, Hubble Space Telescope.

energy by precipitating particles and thermospheric dynamics resulting from corotation breakdown in the equatorial plasma sheet. These processes are responsible for generating ion drifts and joule heating [e.g., Achilleos et al., 1998; Bougher et al., 2005; Millward et al., 2005].

[14] Stallard et al. [2001, 2002] observed an auroral heating event on Jupiter between 8 and 11 September 1998 using the high-resolution IR echelle spectrometer, Cshell, at the NASA Infrared Telescope Facility (IRTF). From this event they extracted information on the Jovian thermospheric winds, temperature, and $\mathrm{H}_{3}^{+}$composition. The derived $\mathrm{H}_{3}^{+}$ temperature rose from 940 to $1065 \mathrm{~K}$ while the $\mathrm{H}_{3}^{+}$column density decreased from 1.80 to $1.55 \times 10^{10} \mathrm{~cm}^{-2}$, suggesting that the energy balance is controlled by $\mathrm{H}_{3}^{+}$cooling. Most recently, Melin et al. [2006] examined the effect of local thermodynamic equilibrium (LTE) on the heating and cooling sources for such an event on the basis of their empirical model. They showed that the departure of excited vibrational levels from LTE occurs at altitudes $>500 \mathrm{~km}$ (above the 1 mbar level), thereby affecting the $\mathrm{H}_{3}^{+}$cooling rates. The higher temperatures observed on 11 September 1998 were attributed to excess energy resulting from an increase in joule heating and ion drag effects.

\subsection{Temperature Structure From UV Emission}

[15] The Jovian FUV auroral spectrum is dominated by the $\mathrm{H}$ Lyman $\alpha$ line and the $\mathrm{H}_{2}$ Lyman $\left(\mathrm{B}^{1} \Sigma_{\mathrm{b}}^{+} \rightarrow \mathrm{X}^{1} \Sigma_{\mathrm{g}}^{+}\right.$) and Werner $\left(\mathrm{C}^{1} \Pi^{+} \rightarrow \mathrm{X}{ }^{1} \Sigma_{\mathrm{g}}^{+}\right)$band systems between 875 and $1600 \AA$ [e.g., Yung et al., 1982; Gladstone and Skinner, 1988; Clarke et al., 1994; Liu and Dalgarno, 1996; Trafton et al., 1998; Ajello et al., 2001, 2005; Gustin et al., 2004a, 2004b, 2006]. Spectral analyses of the $\mathrm{H}_{2}$ bands provide useful constraints on the atmospheric temperature profile through fitting of rovibrational bands and the composition of the atmosphere through absorption of the $\mathrm{H}_{2}$ emissions by overlying hydrocarbons, primarily $\mathrm{CH}_{4}$ and $\mathrm{C}_{2} \mathrm{H}_{2}$. Many groups have been involved in analyzing auroral UV spectra. The study of high-resolution observations $(0.3-0.5 \AA)$ of Jupiter's aurora with the Goddard High-Resolution Spectrograph (GHRS) on board HST has determined a range of rovibrational $\mathrm{H}_{2}$ temperatures of $300-900 \mathrm{~K}$ characterized by a pressure level in the range from 10 to $0.1 \mu$ bar [Clarke et al., 1994; Trafton et al., 1994, 1998; Kim et al., 1997].
Reanalysis of the GHRS HST spectra longward of $1200 \AA$ by Liu and Dalgarno [1996] yielded an effective $\mathrm{H}_{2}$ temperature of $400-600 \mathrm{~K}$ at around $7 \mu$ bar. This range of temperature may be interpreted in terms of temporal variations of the ratio of the soft electron component that excites the EUV $(800-1100 \AA)$ to the hard electron flux that excites the FUV (1100-1700 А) [Ajello et al., 1998, 2001, 2005].

[16] Gustin et al. [2002] derived temperature and composition information from an analysis of spatially resolved FUV spectra (obtained with the Space Telescope Imaging Spectrograph (STIS) on HST) across the main Jovian oval and in the polar cap region of the northern hemisphere. They obtained an effective $\mathrm{H}_{2}$ temperature of about $540 \pm$ $30 \mathrm{~K}$ for the main oval and a lower temperature of about $300 \pm 10 \mathrm{~K}$ for the polar cap region. The temperature of the main oval emission region is located at about $0.7 \mu$ bar, while the polar cap temperature is located deeper in the atmosphere, at about $2 \mu$ bar.

[17] Information on the neutral atmosphere has also been derived from a study of high-resolution $(\sim 0.22 \AA)$ spectra of Jupiter's northern aurora observed in three spectral windows (905-970 $\mathrm{\AA}, 1030-1080 \AA$, and 1090-1180 $\mathrm{\AA}$ ) with the Far Ultraviolet Spectroscopic Explorer (FUSE). Gustin et al. [2004a] reported a comprehensive analysis of the FUSE spectra with many new $\mathrm{H}_{2}$ spectral features that were not accessible before using low-resolution instruments. Since spectra in each window are subject to self-absorption and possible $\mathrm{CH}_{4}$ absorption, the analysis derived $\mathrm{H}_{2}$ temperatures of $850_{-95}^{+90}, 800_{-135}^{+90}$, and $800_{-45}^{+45} \mathrm{~K}$, representing $905-$ $970 \AA, 1030-1080 \AA$, and $1090-1180 \AA$, respectively, with corresponding pressure levels of $0.01_{-0.007}^{+0.02}, 1.20_{-0.52}^{+0.47}$, and $1.04_{-0.45}^{+0.63} \mu$ bar. For the only spectral window in which the $\mathrm{CH}_{4}$ absorption cross section varies substantially with wavelength $(1090-1180 \AA)$, a vertical column of $2.1 \times 10^{15} \mathrm{~cm}^{-2}$ of $\mathrm{CH}_{4}$ was derived corresponding to a pressure of about $2 \mu$ bar [e.g., Gladstone et al., 1996]. Table 1 summarizes Jovian auroral temperatures inferred from existing data sets.

\subsection{High-Latitude Wind Structure}

[18] All existing studies, both theoretical and observational, suggest the presence of strong auroral-driven thermospheric winds with velocities of up to tens of kilometers per second [Sommeria et al., 1995; Achilleos et al., 1998; 
Rego et al., 1999; Stallard et al., 2001; Bougher et al., 2005; Millward et al., 2005]. The need to constrain the modeling of auroral dynamics on Jupiter has led observers to search for a means of measuring such winds using high-resolution spectrometry. While the modeling of asymmetric Ly- $\alpha$ auroral line profiles provides indirect evidence of neutral winds of magnitudes in the range $1-2 \mathrm{~km} \mathrm{~s}^{-1}$ in Jupiter's thermosphere [e.g., Prangé et al., 1997; Gladstone et al., 1998], direct ion wind determinations are possible through measured Doppler shifts of strong $\mathrm{H}_{3}^{+}$emission lines. Rego et al. [1999] presented the first evidence of such winds in Jupiter's northern auroral region from an analysis of highresolution $\mathrm{H}_{3}^{+}$emission lines observed with Cshell on IRTF. Their analysis showed the presence of an anticorotational ion wind of magnitude $>2 \mathrm{~km} \mathrm{~s}^{-1}$ in the main oval ionosphere. Recently, using the same instruments, Stallard et al. [2001, 2002] observed an auroral event on Jupiter to obtain information on $\mathrm{H}_{3}^{+}$winds in addition to $\mathrm{H}_{3}^{+}$temperature and column density in the main oval and polar region. They obtained ion winds somewhat weaker in magnitude than observed by Rego et al. [1999], $0.5 \mathrm{~km} \mathrm{~s}^{-1}$ at the beginning of the event to $1 \mathrm{~km} \mathrm{~s}^{-1}$ at the end of the event. The observed $\mathrm{H}_{3}^{+}$ flows with these velocities were again found to be counter to the planetary rotation around the main oval region, in agreement with Rego et al. [1999].

[19] Plasma flows in Jupiter's middle magnetosphere were measured by Voyager [e.g., Eviatar and Barbosa, 1984; Kane et al., 1995] and Galileo [e.g., Krupp et al., 2001; Frank and Paterson, 2001]. These measurements provide strong evidence of local time-dependent subcorotation of the plasma $(E \times B$ drift) pattern, indicating that electric fields are imposed on the Jovian ionosphere. These fields could drive currents and produce joule heating because of the frictional effect of ions being horizontally accelerated by the electric field through the neutral atmosphere. This mechanism for accelerating ions focuses on corotation breakdown of magnetospheric plasma further away from Jupiter, setting up a current system through the plasma sheet that follows the magnetic field lines down toward the planet and closes in the ionosphere [e.g., Hill, 2001; Cowley and Bunce, 2001; Cowley et al., 2003a, 2003b, 2005; Nichols and Cowley, 2004]. As discussed above, the whole process results in charged particles that accelerate to energies up to tens of $\mathrm{keV}$ from the plasma sheet to the Jovian upper atmosphere, resulting in neutral heating in addition to ionization and auroral emissions. Joule heating combined with particle heating may have great implications in the generating of meridional winds because of the mechanical acceleration of neutrals by ion drag. We have previously shown that such winds with speeds $\geq 125 \mathrm{~m} \mathrm{~s}^{-1}$ [e.g., Bougher et al., 2005; Majeed et al., 2005] are sufficient to transport enough energy to low latitudes from the polar regions to maintain the high exospheric temperature observed at the Jovian equator [Seiff et al., 1998]. These winds also play a significant role in modifying the global thermal budget.

[20] This conclusion is contradictory to a recent study of some of the characteristics of Saturn's neutral winds by Smith et al. [2007]. They showed that the net effect of auroral energy inputs on Saturn's thermosphere is to increase heating in the polar region while cooling the low-latitude region. One possible reason for this difference is that the Smith et al. model is axially symmetric and does not take into account the effects of hemispheric asymmetries discussed above. Another difference between the JTGCM results and those of Smith et al. is their prediction of upwelling at subauroral latitudes, which is opposite to the JTGCM result showing compression of neutral gas as a result of downwelling at these latitudes [Majeed et al., 2005]. Our aim in this paper is to discuss the vertical profiles of thermal balances in JTGCM simulations of Jupiter's auroral region that can best describe the existing temperature data (described above) derived from various sources. A detailed JTGCM wind study is deferred to a subsequent paper.

\section{A Brief Description of the JTGCM}

[21] The major characteristics of the JTGCM have been described by Bougher et al. [2005] in a comprehensive study of the thermospheric dynamics, energetics, and redistribution of thermospheric composition at Jupiter. Here, we briefly describe important points of the JTGCM that are related to this study.

[22] The JTGCM uses a $5^{\circ}$ latitude by $5^{\circ}$ longitude grid with 39 vertical pressure layers in increments of 0.5 pressure-scale heights. The model solves coupled thermodynamic, zonal momentum, meridional momentum, continuity, and hydrostatic equations self-consistently using the basic framework of the National Center for Atmospheric Research (NCAR) general circulation model [cf. Roble et al., 1988]. Each of these equations is cast in log pressure coordinates $\left[Z_{p}=\ln \left(p_{0} / p\right)\right]$, with a specified reference pressure level corresponding approximately to the average homopause level. For the JTGCM code, this reference pressure is located at $4.5 \mu \mathrm{bar}\left(Z_{\mathrm{p}}=0\right)$. Each $Z_{\mathrm{p}}$ interval corresponds to a 1 scale height (at the local temperature).

\subsection{Lower Boundary Conditions}

[23] The lower boundary in the JTGCM is at $20 \mu \mathrm{bar}$, and is used to take into account hydrocarbon cooling due to $\mathrm{C}_{2} \mathrm{H}_{2}(12.6 \mu \mathrm{m})$ and $\mathrm{CH}_{4}(7.8 \mu \mathrm{m})$ at the base of the thermosphere, below the homopause [Drossart et al., 1993]. This is important for proper cooling of the Jovian auroral atmosphere, since electron precipitation provides strong heating that is conducted downward and radiated away via these strong infrared (IR) emissions. $\mathrm{H}_{3}^{+}$cooling from IR emissions [Drossart et al., 1989] is also included above the homopause. Our assumed boundary conditions are that the geopotential, zonal, and meridional winds are zero at the lower boundary (i.e., strict corotation). This is a crude simplification that neglects the strong stratospheric winds [e.g., Flasar et al., 2004] and upward propagating tides and gravity waves [e.g., Young et al., 1997, 2005; Matcheva and Strobel, 1999; Hickey et al., 2000] that must be present in the Jovian lower atmosphere. Global average lower boundary conditions both for the temperature and neutral densities $(\mathrm{H}$ and $\mathrm{He})$ are taken from Galileo [Seiff et al., 1998] and Voyager data [Festou et al., 1981]. Specifically, an average global temperature, composed of observed equatorial and polar values near $250 \mathrm{~km}$, is set to $190 \mathrm{~K}$. The helium volume mixing ratio is set to 0.135 at $250 \mathrm{~km}$ or $20 \mu \mathrm{bar}$, on the basis of Galileo probe observations [Niemann et al., 1996]. The atomic hydrogen volume mixing ratio is set to $4.23 \times 10^{-8}$ at the lower boundary, in accord with Voyager 
data [Gladstone et al., 1996]. Photochemical equilibrium is assumed for the major ions $\left(\mathrm{H}_{2}^{+}\right.$and $\left.\mathrm{H}_{3}^{+}\right)$.

\subsection{Upper Boundary Conditions}

[24] Upper boundary conditions are specified at about $1.1 \times 10^{-4}$ nbar in order to properly include high-altitude auroral heating processes [Ajello et al., 2001; Grodent et al., 2001; Gustin et al., 2004a, 2004b] and $\mathrm{H}_{3}^{+}$cooling in the near-IR [Drossart et al., 1989]. Corresponding boundary conditions for temperatures and neutral winds are identical to those employed in the terrestrial TIGCM; i.e., vertical gradients in temperatures and winds (zonal, meridional, and vertical) are set to zero at the top of the model. These conditions are in accord with weak energy sources at the highest altitudes; isothermal temperatures are also consistent with the emergence of the exosphere. For composition $(\mathrm{H}$ and $\mathrm{He}$ ), diffusive equilibrium is assumed at the top boundary [cf. Bougher et al., 2005].

[25] Each of the JTGCM equations is time dependent, but is typically integrated toward steady state conditions. The JTGCM simulations of the thermosphere/ionosphere are run for many Jovian rotations in order to achieve a steady state solution in the modeled fields. Planetary TGCMs achieve steady state solutions according to various timescales that vary as a function of altitude [e.g., Bougher et al., 1999]. For Jupiter's thermosphere, a useful dynamical timescale can be defined as the transport time for average meridional winds to redistribute auroral oval heating and atomic species to the jovigraphic equator. Typical JTGCM zonally averaged meridional winds $\left(125-300 \mathrm{~m} \mathrm{sec}^{-1}\right)$ [Bougher et al., 2005] place this timescale at about 4 to 10 Earth days for pressures less than about $0.15 \mu$ bar. Equilibration requires that meridional pressure gradients are stabilized by both pole-to-equator and equator-to-pole wind flows, for which the dynamical timescales should be multiplied by $\sim 2$. This yields an effective dynamical timescale of the order of 8-20 Earth days (or 20-50 Jovian rotations). Hence, JTGCM calculations can only achieve near-equilibrium solutions for the upper thermosphere ( $p<0.15 \mu$ bar) when simulations run on the order of $>50$ Jovian rotations. Further discussion on the JTGCM computational scheme, numerical stability, filtering and smoothing of prognostic fields is presented by Bougher et al. [2005].

\subsection{Input Parameters}

[26] The JTGCM uses solar EUV radiation as a source of equatorial and midlatitude heating, while the particle heating calculated by Grodent et al. [2001], on the basis of their onedimensional electron transport and thermal transport models, is prescribed for the auroral region. An average solar EUV heating rate profile estimated by Waite et al. [1983] is specified within the latitude band of $-50^{\circ}$ to $+50^{\circ}$ latitude.

[27] The auroral heating calculation from Grodent et al. [2001] prescribed for the two Jovian ovals uses an incident electron energy spectrum described by a combination of the following three Maxwellian distributions: (1) $22 \mathrm{keV}$ particles (100 ergs cm $\left.\mathrm{cm}^{-2} \mathrm{~s}^{-1}\right)$, (2) $3 \mathrm{keV}$ particles $\left(10 \mathrm{erg} \mathrm{cm}^{-2} \mathrm{~s}^{-1}\right)$, and (3) $100 \mathrm{eV}$ particles $\left(0.5 \mathrm{ergs} \mathrm{cm}^{-2} \mathrm{~s}^{-1}\right)$. For each of these components, energy is roughly deposited near the homopause, just above the homopause, and high in Jupiter's thermosphere, respectively. The total auroral energy flux is
110 ergs $\mathrm{cm}^{-2} \mathrm{~s}^{-1}\left(1 \mathrm{erg} \mathrm{cm}^{-2} \mathrm{~s}^{-1}=1 \mathrm{~mW} \mathrm{~m}^{-2}\right.$ for readers preferring SI units). The choice of such an energy spectrum allowed Grodent et al. [2001] to reproduce the observed emission of a discrete aurora and to evaluate thermal balance in comparison with derived temperature data from remotesensing observations of Jupiter's aurora. It is, however, important to note that typical main auroral oval intensities are well established to be a few hundred $\mathrm{kR}$, thus indicating a typical auroral electron energy flux of a few tens of ergs cm $\mathrm{cm}^{-2} \mathrm{~s}^{-1}$ [cf. Cowley et al., 2005]. Following Grodent et al. [2001], there have been several studies [e.g., Ajello et al., 2001, 2005; Gustin et al., 2002, 2004a, 2004b, 2006; Gérard et al., 2003] using electron energy distribution functions with initial electron energy $\left(\mathrm{E}_{\mathrm{o}}\right)$ in the range 15$230 \mathrm{keV}$ and associated energy fluxes roughly in the range $1-278$ ergs $\mathrm{cm}^{-2} \mathrm{~s}^{-1}$ to explain observed characteristics of the UV spectra. This implies that there are a very wide range of values used to interpret the measured auroral brightness and the FUV color ratio (CR) of auroral emissions emanating from different regions of the main auroral oval and the polar cap. Some of these studies have also inferred information on the background neutral thermal structure of the auroral thermosphere. However, a recent study by Gustin et al. [2006] has shown that HST-STIS spectra require mean energy flux $\left(\Phi_{\mathrm{o}}\right)$ in the range $4.7-90.4 \mathrm{ergs} \mathrm{cm}^{-2} \mathrm{~s}^{-1}$ to explain the intensity of auroral emissions and FUV CR of four different regions of the northern oval without deriving information on the processes of the background thermal structure. Instead, they employed Knight's theory [Knight, 1973] to quantify field-aligned currents flowing in these four oval regions from a relationship between FUV CR and the mean energy of the precipitating electrons $(\langle E\rangle)$. Because FUV CR is a measure of the absorption of the $\mathrm{H}_{2}$ auroral emission by hydrocarbons within or above the region of auroral energy deposition, it can be used to provide an indirect measure of the mean energy of the precipitating electrons. The relationship between FUV CR and the electron mean energy depends on the altitude distribution of the hydrocarbons [see Gérard et al., 2002], which can be determined using the method described by Grodent et al. [2001]. A given primary electron distribution function at the top of an assumed model atmosphere with an assumed value of the eddy diffusion coefficient at the homopause $\left(\sim 10^{6} \mathrm{~cm}^{-2} \mathrm{~s}^{-1}\right)$ will result in a particular FUV CR. Since a different eddy diffusion coefficient would result in a different FUV CR in regions of enhanced particle precipitation and heating such as the main auroral oval [e.g., Livengood et al., 1990], the relationship between CR and $\langle\mathrm{E}\rangle$ must be modified [e.g., Gérard et al., 2002]. Consequently, the background neutral thermal structure of the auroral thermosphere must also be adjusted. A detailed calculation of these effects on the oval emission and on the energy budget of the neutral atmosphere would require knowledge of a three-dimensional time-dependent distribution of auroral energy inputs, which is not possible with current data and models.

[28] The energy distribution function used by Grodent et al. [2001] to calculate the altitude profile of the auroral heating rates is in the range mentioned above and is a reasonable starting point to simulate steady state temperature profiles and to quantify the auroral energy budget for the 
Jovian thermosphere for comparison with existing temperature data derived from a variety of different experiments designed to probe different regions of Jupiter's aurora.

[29] The locations of the polar ovals are currently described by the auroral morphology deduced from analysis of WFPC2 images taken in 1996 and 1997 [Clarke et al., 1996, 1998] and recently by HST-STIS images [Grodent et al., 2003]. These analyses show that the general shape of the main auroral ovals and their locations in the two hemispheres remain approximately constant in latitude and system III longitude over many years of UV observations. However, in the most recent study of HST-STIS images, the brightness of the main auroral oval has been questioned. Grodent et al. [2008] have shown that the main oval is the major contributor (almost 75\%) of the Jovian auroral brightness integrated over the pole. The local time variation of this brightness has led Grodent et al. [2008] to reveal several individual details of the characteristics of the main auroral oval in terms of local time. They showed that a relatively narrow arc of continuous UV emission represents a fraction of the main oval in the dawn-to-noon sector, while the noonto-dusk sector contained auroral patches that appeared to broaden and break from the main oval. Because of this continuity, Grodent et al. [2008] substituted the term "main oval" with the term "main emission." Even with this new terminology, we prefer to use the term "main oval" in the rest of the paper to describe the term "main emission."

[30] Note that the specified particle heating profile as a function of pressure is identical everywhere in the JTGCM ovals. Note also that the oval width is limited to $5^{\circ}$ latitudelongitude resolution of the JTGCM code, effectively yielding a delta function for auroral forcing along the two ovals. The oval width in the JTGCM is coarser than that of Jupiter's real oval; nevertheless, the integrated energy flux is assumed to be the same. In addition, joule heating, which is driven by the differential velocity between the ion and neutral species in the auroral ionosphere, has also been described in the JTGCM code as an important mechanism for modifying Jupiter's global thermospheric winds and temperatures. The treatment of joule heating in the JTGCM is described later.

[31] An important parameterization in the JTGCM is the high-latitude ion drift around the auroral ovals resulting from the magnetic coupling between an equatorial plasma sheet within the middle magnetosphere and the Jovian upper atmosphere. As described above, the main auroral ovals are linked to the breakdown of plasma sheet corotation at 20$30 \mathrm{Rj}$, where large field-aligned currents are generated by the transfer of Jupiter's angular momentum to iogenic plasma that is being driven centrifugally outward [Cowley et al., 2005, and references therein]. This process results in the precipitation of energetic electrons, causing ionization and heating of the neutral gas. Thus, the main ovals are a dominant source of much of the auroral ionization, producing a highly conducting ionosphere, and are the likely origin of fast ion drifts in Jupiter's ionosphere - the auroral electrojet [e.g., Cowley and Bunce, 2001]. For these calculations, we use an estimated convection electric field and corresponding ion drifts $\left(\mathrm{u}_{\mathrm{i}}\right.$ and $\left.\mathrm{v}_{\mathrm{i}}\right)$ from a simplified ionospheric convection model as inputs. Such estimates are based on Voyager measurements of ion convection in the outer magnetosphere [cf. Eviatar and Barbosa, 1984] mapped to high latitudes using the VIP4 magnetic field model [Connerney et al.,
1998]. In this manner, anticorotational electrojet winds up to $3.0 \mathrm{~km} \mathrm{~s}^{-1}$ (see Bougher et al. [2005] for details) are estimated and prescribed around both main auroral ovals, driving the neutral winds to move in the same direction. Conversely, in nonauroral regions, $\mathrm{u}_{\mathrm{i}}$ and $\mathrm{v}_{\mathrm{i}}$ are zero (i.e., corotational).

[32] It is important to note that Voyager flyby missions were designed to measure the energetic plasma distribution in Jupiter's magnetosphere along their trajectories for a few days. These measurements showed that energetic ions and plasma are essentially corotating with the planet because of a convective motion of ions around the planet together with the plasma concentrated near the magnetic equatorial plane [e.g., Carbary et al., 1981]. However, as mentioned above, the breakdown of such a magnetospheric plasma corotation has been found to be responsible for the measured brightness of Jupiter's main auroral oval emission. In an empirical model, Eviatar and Barbosa [1984] determined the plasma flow to be in the range $200-500 \mathrm{~km} \mathrm{~s}^{-1}$ for a corresponding radial distance in the range $16-50 R j$. These estimates are in reasonably good agreement with the measured plasma flow determined by Krupp et al. [2001] from the much expanded Galileo orbiter data for the Jovian equatorial plane.

[33] Currently, we are in the process of developing a selfconsistent coupled atmospheric-thermospheric-magnetospheric model to study the Jovian system using the JTGCM, Galileo in situ measurements of the ion convection and field-aligned currents, Earth-based observations of the aurora at IR, UV, and X-ray wavelengths, a magnetohydrodynamic (MHD) model of Jupiter's magnetospheric dynamics with full interaction with the solar wind, and a full wave model to parametrically specify the upward propagating gravity wave spectrum. This will allow us to address feedbacks between the thermosphere, atmosphere, and magnetosphere, in addition to getting information on the extent the global magnetospheric dynamics and global thermospheric dynamics affect this coupling. The coupled model will also be used to address the transport of atmospheric angular momentum vertically to the region of maximum joule heating and Pedersen conductivity in the Jovian thermosphere. The results presented in this paper are therefore critically important because they provide a useful benchmark for future modeling of the Jovian system.

[34] Ion drag within the JTGCM code is described as a dominant physical process, which limits neutral wind speeds through coupling between ions in the Jovian auroral ovals and the corotating neutral atmosphere. The ions, magnetically connected to the subcorotating regions of the magnetosphere, lose their momentum in collisions with neutrals and drive the neutrals to move in roughly the same direction. In the auroral region, this drag is proportional to the product of the ion density (mostly $\mathrm{H}_{3}^{+}$) and the relative drift speed between the neutral and ion constituents. The density of $\mathrm{H}_{3}^{+}$in the JTGCM is calculated assuming photochemical equilibrium owing to its short lifetime $\left(<10^{3} \mathrm{~s}\right)$. A parameterized function of $\mathrm{H}_{2}^{+}$production [Waite et al., 1983] is assumed to be the dominant source of the $\mathrm{H}_{3}^{+}$ions, while dissociative recombination is considered to be their major sink. The $\mathrm{H}^{+}$ion is presently prescribed within the JTGCM on the basis of a detailed 1-D profile calculated offline [Waite et al., 1983] for all JTGCM locations. The ionosphere is largely dominated by $\mathrm{H}^{+}$production above an altitude of $1200 \mathrm{~km}$ 
and by $\mathrm{H}_{3}^{+}$below this altitude, regardless of the presence of the neutral winds and energetic particle precipitation [Majeed et al., 1999; Maurellis and Cravens, 2001]. Current interpretation of the measured vertical ionospheric structure indicates that a combination of vertical plasma drift and enhanced populations of vibrationally excited $\mathrm{H}_{2}$ molecules with $\nu \geq 4$ is required for fitting the observations with $1-\mathrm{D}$ models [Majeed et al., 2004]. Thus, a proper calculation of $\mathrm{H}^{+}$densities requires a loss mechanism involving $\mathrm{H}_{2}$ vibrational levels [Cravens, 1987; Majeed and McConnell, 1991]. However, this key loss for $\mathrm{H}^{+}$is not properly treated within the Waite et al. 1-D model, and likewise is ignored in the present JTGCM code. The proper calculation of these $\mathrm{H}_{2}$ vibrational levels in a future version of the JTGCM code will allow us to simulate the upper ionosphere selfconsistently with the Jovian thermal and dynamical structures on a global basis.

[35] Ion drag is a dominant neutral momentum forcing process at auroral latitudes near the altitude of the ionospheric peak. At greater ionospheric heights the effect of ion drag gradually decreases as the ion gyrofrequency that constrains ions to move along magnetic field lines exceeds the ion neutral collision frequency. Richmond et al. [1992] have shown that ion drag can significantly modify the neutral winds at Earth's low and midlatitudes, thereby affecting the distribution of neutral temperatures.

[36] The parametrization of ion drag and joule heating in the JTGCM code is based on the formulation described by Dickinson et al. [1984] and Roble and Ridley [1987]. Recently, Bougher et al. [2005] demonstrated that a scaling scheme of ion drag and corresponding joule heating by adjusting the horizontal ion drift can be used to explore general properties of Jupiter's global thermal structure and dynamics, which can then be used to explain the major characteristics of the multispectral observations of the Jovian thermosphere. They suggested a downward scaling of ion drift by $30 \%$ is needed for the JTGCM simulations to best describe the Jovian observations. This scaling likely reflects uncertainties in the (1) magnetosphere-ionosphere mapping that we have conducted using the VIP4 magnetic field model [Connerney et al., 1998] and (2) derived high-latitude ion convection [Eviatar and Barbosa, 1984] from Voyager observations of the middle magnetosphere of Jupiter. It is important to note that we used a different scaling method than that adopted by Bougher et al. [2005]. Rather than adjusting ion drifts, we follow Majeed et al. [2005], who scaled the total joule heating produced in the Jovian auroral ovals by 0.15 in order to reproduce the equatorial thermal structure observed by the Galileo probe. Note that early versions of the Earth TGCM allowed joule heating rates to vary by as much as a factor of 20 from the global heating estimates based on derived time mean currents in the dynamo region [Matsushita et al., 1973], and global simulations from these models were utilized to interpret thermospheric temperatures and radar observations of neutral winds at low and midlatitudes [Dickinson et al., 1975]. Joule heating in current Earth models (e.g., TIEGCM) is induced by high-latitude plasma drifts associated with magnetospheric convection driven by a cross-tail potential that is highly variable, ranging from $20 \mathrm{kV}$ to perhaps $200 \mathrm{kV}$ depending on geomagnetic conditions [Roble and Ridley, 1994]. For the terrestrial thermosphere, this is an important parameter to account for in estimating diurnal neutral temperature distribution and thermospheric circulation. Thus, regardless of which method is to be used, the scaling down of the estimated joule heating is important for modifying the global thermospheric circulation on Jupiter in order to match observations, and this is in accord with early studies with terrestrial TCGMs [cf. Dickinson et al., 1975].

\subsection{Estimates of Hydrocarbon Cooling}

[37] Our estimates of hydrocarbon cooling due to strong $\mathrm{C}_{2} \mathrm{H}_{2}(12.6 \mu \mathrm{m})$ and $\mathrm{CH}_{4}(7.8 \mu \mathrm{m})$ radiation in the JTGCM are based on constraints provided by reanalyzing Voyager 1 infrared Interferometer and Radiometer Spectrometer (IRIS) spectra [Drossart et al., 1993]. Note that the IR cooling by $\mathrm{C}_{2} \mathrm{H}_{6}$ emission is negligible in our JTGCM domain, and is therefore neglected. The total measured excess infrared auroral zone emission (averaged over the IRIS field of view) in the hydrocarbon bands between 7 and $13 \mu \mathrm{m}$ was found to be about $208 \mathrm{erg} \mathrm{cm}^{-2} \mathrm{~s}^{-1}$ over an area of about $2 \times$ $10^{18} \mathrm{~cm}^{2}$ with a resulting power output of $4 \times 10^{13} \mathrm{~W}$. This large IR output likely results from a large temperature enhancement in the upper stratosphere and lower thermosphere, in accord with strong auroral and joule heating that is conducted downward and made available for IR radiation [Bougher et al., 2005].

[38] Yelle et al. [2001] reevaluated radiative processes in Jupiter's stratosphere between 0.1 bar and $10^{-6}$ bar on the basis of constraints provided by the Galileo temperature profile and composition profiles derived from various experiments. Heating and cooling rates were calculated on the basis of realistic altitude profiles of $\mathrm{C}_{2} \mathrm{H}_{2}, \mathrm{C}_{2} \mathrm{H}_{6}$, and $\mathrm{CH}_{4}$, which were in reasonably good agreement with predictions from photochemical models [e.g., Gladstone et al., 1996]. Interestingly, Yelle et al. [2001] predicted that absorption of solar radiation in $\mathrm{CH}_{4}$ bands is the dominant heat source in the equatorial Jovian stratosphere. The $3.3-\mu \mathrm{m}$ band is the primary heat source at pressures less than $4 \times 10^{-6}$ bar, while the $2.3-\mu \mathrm{m}$ band dominates the heat budget between $4 \times 10^{-6}$ and $5 \times 10^{-4}$ bar. Another important conclusion of Yelle et al.'s model is the dominance of $\mathrm{C}_{2} \mathrm{H}_{6}$ cooling throughout the stratosphere with a minor contribution from $\mathrm{C}_{2} \mathrm{H}_{2}$, which is contrary to many earlier studies [e.g., Grodent et al., 2001]. As noted above, the current version of the JTGCM code does not include these heating and $\mathrm{C}_{2} \mathrm{H}_{6}$ cooling terms. Future upgrades to the JTGCM code will incorporate the Yelle et al. model to improve the selfconsistency of the energetic, dynamical, and chemical processes at lower thermospheric altitudes ( $>1 \mu \mathrm{bar})$.

\section{JTGCM Temperature Simulations}

[39] In Figure 1a we show the distribution of neutral temperatures with superimposed horizontal wind vectors near the top boundary of the JTGCM in the Jovian exosphere. This simulation includes auroral forcing by charged particle heating and an additional forcing by $15 \%$ of the total joule heating calculated for the auroral ovals. The simulation was run for 84 Jovian rotations to achieve steady state temperature and neutral wind fields for pressures $<1 \mu$ bar, while at higher pressures the JTGCM fields remain to be stabilized. Note that the thermospheric wind system in both northern and southern auroral regions is largely driven by the 

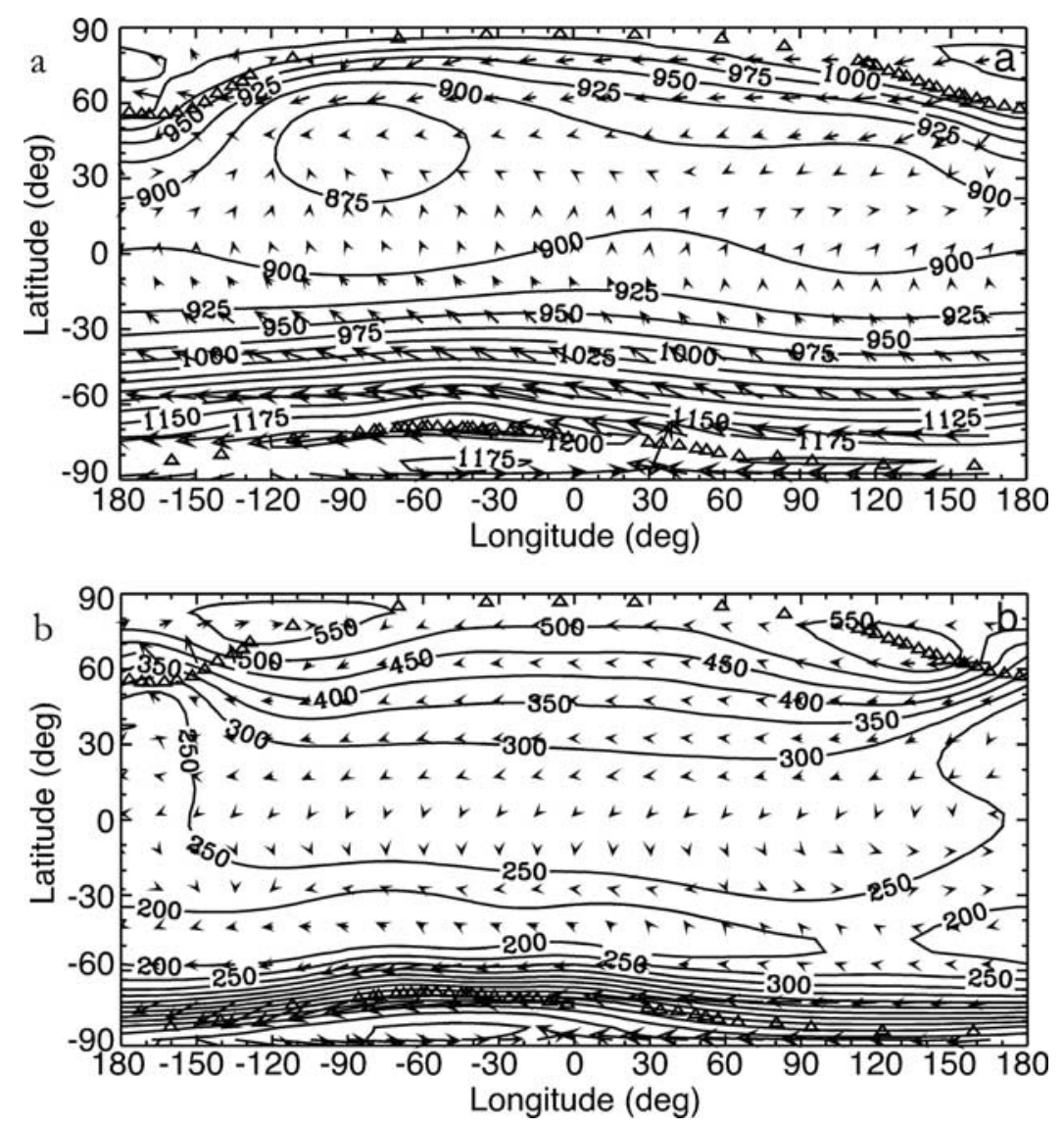

Figure 1. JTGCM simulation showing temperatures with superimposed wind vectors. (a) Pressure level slice near the top of the atmosphere $\left(10^{-4}\right.$ nbar). (b) Pressure level slice near the peak of the $\mathrm{H}_{3}^{+}$ion (about $0.14 \mu$ bar). Maximum horizontal winds range from $1.5 \mathrm{~km} \mathrm{~s}^{-1}$ (Figure 1a) to $2.0 \mathrm{~km} \mathrm{~s}^{-1}$ (Figure 1b). Wind vector lengths are independently scaled to the maximum vector wind speeds for Figures $1 \mathrm{a}$ and $1 \mathrm{~b}$.

imposed high-latitude ion winds [e.g., Bougher et al., 2005] and large pressure gradients. Strong upwelling and divergence of the neutral winds dominate the oval regions (in the south and north) while subsidence and convergence of these winds occurs at the Jovian equator. A maximum neutral wind speed of about $1.8 \mathrm{~km} \mathrm{~s}^{-1}$ is reached in the southern oval while a smaller top wind speed of $\sim 1.0 \mathrm{~km} \mathrm{~s}^{-1}$ is sustained in the northern oval. These diverging thermospheric winds are effective in cooling down the auroral regions. The resulting maximum exospheric temperatures in the northern and southern ovals are $\sim 1000$ and $1200 \mathrm{~K}$, respectively. However, in the polar cap region (latitudes poleward of the main oval), the neutral temperature varies both in the south and north depending on the magnitude of the horizontal neutral winds. A maximum temperature of $1175 \mathrm{~K}$ is found for the southern polar cap region, while a maximum temperature of $1000 \mathrm{~K}$ is present in the northern polar cap region. It is important to note that thermospheric circulation of neutrals has a great impact on the Jovian equatorial temperature distribution. Where this thermospheric flow subsides at low latitudes, acceleration of the neutral wind along the Jovian equator occurs as a result of strong pressure gradient and Coriolis torques. This results in an equatorial temperature of about $900 \mathrm{~K}$, indicating that energy has been transported over the entire planet from the auroral regions. This value is consistent with that observed by the Galileo probe [e.g, Seiff et al., 1998] and recently interpreted by Majeed et al. [2005].

[40] Figure $1 \mathrm{~b}$ illustrates an interesting situation at around $0.14 \mu$ bar $(\sim 500 \mathrm{~km}$ altitude $)$ near the $\mathrm{H}_{3}^{+}$peak. At this pressure, the high-latitude heating rates due to particle precipitation and imposed ion drifts by the convection electric field on the polar ionosphere are sufficiently large to generate a global thermospheric circulation of the neutral flow with speeds up to $1.9 \mathrm{~km} \mathrm{~s}^{-1}$ and $1.2 \mathrm{~km} \mathrm{~s}^{-1}$ in the southern and northern oval regions, respectively. With these magnitudes of neutral winds and the peak electron density (mostly $\mathrm{H}_{3}^{+}$) of 3 to $5 \times 10^{5} \mathrm{~cm}^{-3}$ [cf. Bougher et al., 2005; Kim et al., 1992], the ion neutral collision in the presence of a strong magnetic field enhances the effect of ion drag on the thermospheric flow. Such an effect can be seen in the northern hemisphere, which yields a maximum temperature of $\sim 550 \mathrm{~K}$ in the oval. The distribution of neutral temperatures in the southern hemisphere, on the other hand, is quite variable because the neutral flow is modified by large ion convection velocities. Since the drifting ions accelerate neutrals horizontally by ion neutral collisions, ion drag acts as a driving force for establishing a strong thermospheric flow that can be seen near $-70^{\circ}$ latitude and $-60^{\circ}$ longitude along the southern oval with a maximum temperature of $\sim 600 \mathrm{~K}$. 
[41] Figure $1 \mathrm{~b}$ also shows the distinct single-cell pattern of strong neutral winds that occurs between $60^{\circ}$ and $90^{\circ}$ latitude in both hemispheres. In the southern oval region, a counterclockwise wind pattern emerges, with net vector winds reaching nearly $1.9 \mathrm{~km} \mathrm{~s}^{-1}$. Zonal neutral winds display a very strong westward jet (up to $-1.0 \mathrm{~km} \mathrm{~s}^{-1}$ ) at high southern latitudes, with a return flow (up to $0.8 \mathrm{~km} \mathrm{~s}^{-1}$ ) closer to the pole. The corresponding meridional neutral winds are equatorward (up to $\sim 0.7 \mathrm{~km} \mathrm{~s}^{-1}$ ) and poleward (up to $-0.7 \mathrm{~km} \mathrm{~s}^{-1}$ ). The net result is a single-cell (anticorotational) neutral wind pattern that is driven by the prescribed ion winds (up to $3 \mathrm{~km} \mathrm{~s}^{-1}$ ) illustrated in Figure 8 of Bougher et al. [2005]. The neutral wind pattern is also very active in the region poleward of the southern ovals. This results in a maximum temperature of $750 \mathrm{~K}$ in the southern polar cap region. Similarly, in the northern oval region, a clockwise single-cell (anticorotational) neutral wind pattern is visible that responds closely to the electrojet in the northern hemisphere. However, the neutral wind speeds in the northern oval region are about $50-60 \%$ of the corresponding values of the southern oval region. This explains the lower maximum temperature of $\sim 550 \mathrm{~K}$ simulated by the JTGCM for the northern oval and polar cap regions. One possible explanation of this asymmetry is that ion drag is more effective in the southern auroral oval region, in part due to the $\sim 20 \%$ larger maximum vector ion winds that are mapped from the magnetosphere to the ionosphere [Bougher et al., 2005; see also Millward et al., 2005]. In addition, the local topology of the VIP4 magnetic field in the northern hemisphere is much different from that in the south [cf. Connerney et al., 1998]. This suggests that the Pedersen conductivities are generally larger in the southern hemisphere than in the northern hemisphere. JTGCM diagnostics reveal a 30-40\% larger column-integrated Pedersen conductivity $\left(\Sigma_{\mathrm{p}}\right)$ in the southern oval region compared to that in the northern oval region [see Bougher et al., 2005]. Finally, a geometric effect occurs for which the spatially larger southern hemisphere drift pattern gives rise to ion drag forcing (and joule heating) that is continuously applied to the southern auroral oval region over a large fraction of a Jovian day. Overall, these features determine that the enhanced ion drag and the associated stronger joule heating combine to yield stronger neutral winds in the southern auroral region compared to those in the northern auroral region. In general, it is clear that ion drag and joule heating inputs have significantly modified the neutral wind patterns and temperature distributions in both the northern and southern oval and polar cap regions.

[42] We have also estimated column-integrated Pedersen conductivities $\left(\Sigma_{\mathrm{p}}\right)$ in the oval ionosphere. An average value of $\Sigma_{\mathrm{p}}$ for the southern oval has been estimated to be $\sim 20$ mhos, consistent with Bougher et al. [2005]. This value has been reduced to about 12 mhos for the northern oval owing to asymmetric neutral flow in the two hemispheres, as discussed above. While a direct comparison between our estimates of $\Sigma_{\mathrm{p}}$ and available $\Sigma_{\mathrm{p}}$ calculated by two different models [Millward et al., 2002, 2005; Cowley et al., 2005] cannot be made because of the use of different auroral parameters, a rough comparison is possible where applicable.

[43] Millward et al. [2002] investigated how the auroral ionosphere and its conductivity respond to variations in the auroral energy and associated energy flux of the precipitating electrons within the Jovian oval. For a constant precipitating electron energy flux of $10 \mathrm{erg} \mathrm{cm}^{-2} \mathrm{~s}^{-1}$, they calculated $\Sigma_{\mathrm{p}}$ equals 0.75 and 1.75 mhos for $30 \mathrm{keV}$ and $60 \mathrm{keV}$ electrons, respectively. Millward et al. [2002] have also investigated how auroral conductivities are affected by increasing electron energies for a constant electron number flux of $6.25 \times 10^{8} \mathrm{~cm}^{-2} \mathrm{~s}^{-1}$ (not an energy flux). A value of $\Sigma_{\mathrm{p}}$ equaling 1.8 mhos was calculated for precipitating electron energies of 30 and $80 \mathrm{keV}$. Interestingly, they obtained a maximum $\Sigma_{\mathrm{p}}$ of nearly 8 mhos for the electron energy of $60 \mathrm{keV}$, suggesting that this energy is capable of producing an ionosphere with a peak density of $3 \times 10^{6} \mathrm{~cm}^{-3}$ within the Pedersen layer to control the integrated conductivities. In a later paper, Millward et al. [2005] modified JIM by including a transauroral voltage up to $3 \mathrm{MV}$ (equivalent to an electric field of $0.6 \mathrm{~V} \mathrm{~m}^{-1}$ ) to simulate the electric field resulting from corotation lag of the plasma sheet. Using this modified version of JIM, they calculated the dependence of $\Sigma_{\mathrm{p}}$ on electron number flux for the precipitating electron energy of $30 \mathrm{keV}$ in the northern oval at $58^{\circ} \mathrm{N}, 180^{\circ} \mathrm{E}$. They showed that $\Sigma_{\mathrm{p}}$ increases linearly from 0.1 to more than 10 mhos for a corresponding increase in electron number flux from $2 \times 10^{7} \mathrm{~cm}^{-2} \mathrm{~s}^{-1}$ to $2 \times$ $10^{10} \mathrm{~cm}^{-2} \mathrm{~s}^{-1}$, respectively. This linear dependence of $\Sigma_{\mathrm{p}}$ on the precipitating electron flux allows us to compare the JTGCM estimates of Pedersen conductivity with the JIM cases. For the JIM case of electron energy of $30 \mathrm{keV}$ and electron number flux of $6.25 \times 10^{8} \mathrm{~cm}^{-2} \mathrm{~s}^{-1}$ [e.g., Millward et al., 2002], it turns out that the precipitating electron energy flux is $\sim 30 \mathrm{ergs} \mathrm{cm}^{-2} \mathrm{~s}^{-1}$, about a factor of seven too small compared to the JTGCM prescription of auroral flux. For this case, the JTGCM estimate of $\Sigma_{\mathrm{p}}$ for the northern oval is about a factor of seven larger than the JIM case. However, a comparison between the JTGCM and the modified version of JIM [e.g., Millward et al., 2005, Figure 11] indicates that the JTGCM auroral inputs $\left(\mathrm{E}_{\mathrm{o}}=\right.$

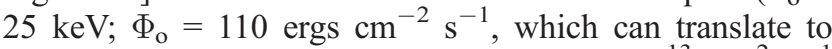
an electron number flux equal to $2.75 \times 10^{13} \mathrm{~m}^{-2} \mathrm{~s}^{-1}$ for $25 \mathrm{keV}$ electrons) should predict a value of $\Sigma_{\mathrm{p}}$ nearly a factor of three larger than that calculated by JIM. These JIM and JTGCM differences in $\Sigma_{\mathrm{p}}$ may be attributed to several factors including (1) inputs for auroral electron precipitation, (2) magnetic field models, (3) plasma drift velocities, and (4) integration times of the two models. For instance, the run time for JIM simulations is about a few Jovian days, while the JTGCM simulations are integrated for 85 Jovian days to achieve steady state solutions for temperatures and three-component neutral wind fields.

[44] Cowley et al. [2005] using an empirical model of magnetosphere-ionosphere coupling currents, with auroral inputs from Millward et al. [2002, 2005], found that variations in $\Sigma_{\mathrm{p}}$ depend both on precipitating electron flux and electron energy, which in turn depend on magnetospheric source populations. They showed that the effective $\Sigma_{\mathrm{p}}$ increases [Cowley et al., 2005, Figure 5] in response to the source population in the middle magnetosphere as the field-aligned current increases up to $0.6 \mu \mathrm{A} \mathrm{m}^{-2}$. As estimated above, the JTGCM electron number flux of $2.75 \times$ $10^{13} \mathrm{~m}^{-2} \mathrm{~s}^{-1}$ can be multiplied by the electron charge to yield a field-aligned current of roughly $4.4 \mu \mathrm{A} \mathrm{m}^{-2}$. Clearly, this value is approximately an order of magnitude larger 

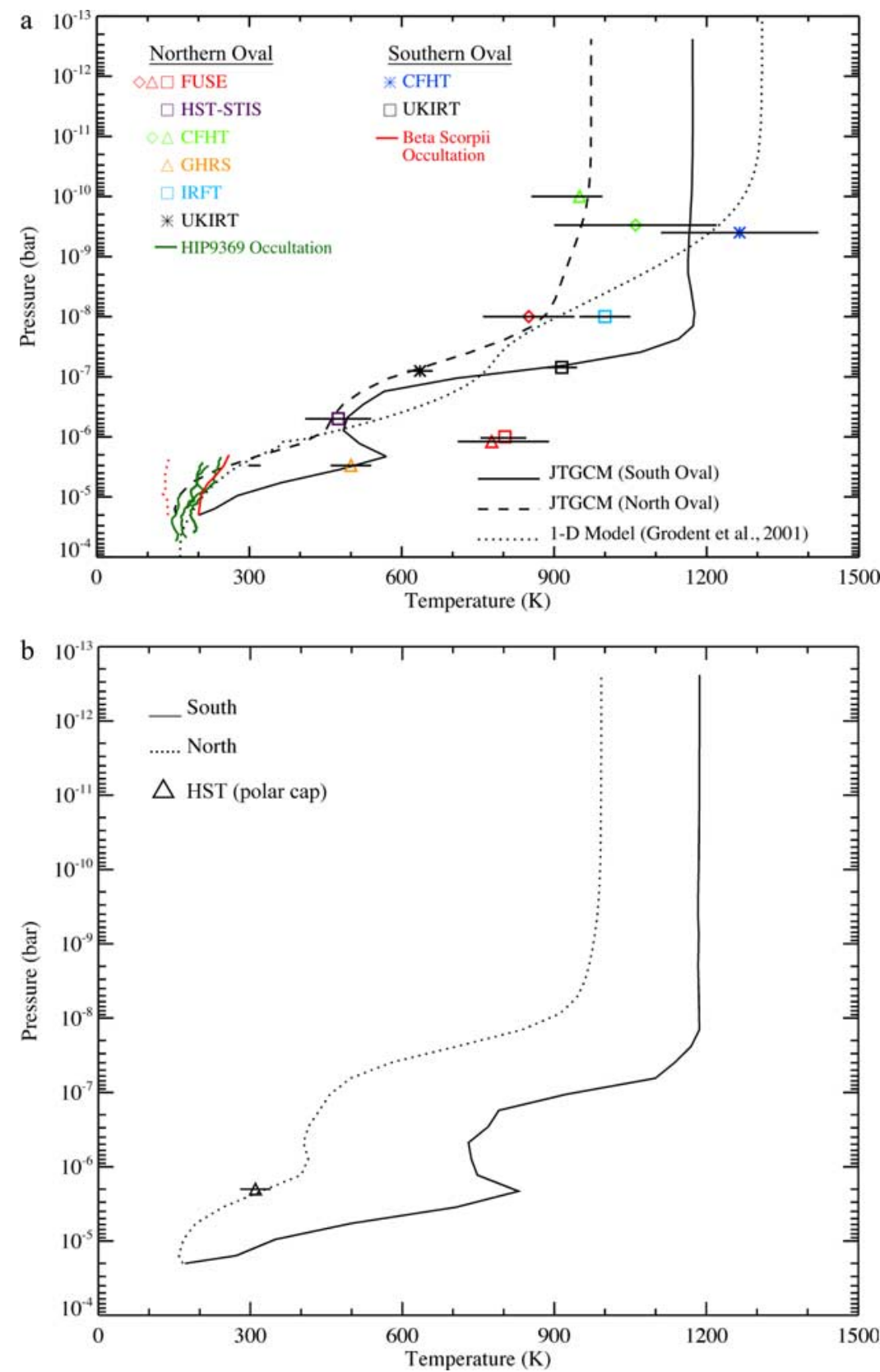

Figure 2. (a) Average JTGCM temperature profiles both for southern and northern auroral ovals are shown in comparison with remotely sensed temperature observations from various sources. This simulation incorporates $15 \%$ of the total joule heating produced in auroral ovals. (b) Same as Figure 2a but for the region poleward of the auroral ovals.

than Cowley et al.'s value of $0.4 \mu \mathrm{A} \mathrm{m}^{-2}$ for which $\Sigma_{\mathrm{p}}$ is about 2.6 mhos (corrected for $1-\mathrm{K}$ factor, where $\mathrm{K}$ is assumed to be 0.5 by Cowley et al.). This value of $\Sigma_{\mathrm{p}}$ is about a factor of 4 and 7 smaller than that estimated by the JTGCM for the northern and southern oval regions, respectively.

\section{Comparison With Auroral Temperature Data}

[45] In Figure 2 we compare JTGCM temperature profiles, simulated for the main auroral ovals and polar cap regions for the two Jovian hemispheres, with measured and modeled thermal structures from various sources listed in Table 1. For simulated oval profiles, the JTGCM incorporates ion drag terms and $15 \%$ of the total joule heating produced in the ovals, in addition to auroral heating caused by precipitated charged particles. Since no charged particle heating is prescribed for the polar cap regions, only ion drag and joule heating ( $15 \%$ efficiency) terms are used to simulate temperatures for those regions. The implications of fractional joule heating have been discussed in detail by Bougher et al. [2005] with respect to the main characteristics of the Jovian thermosphere/ionosphere system including global temper- 
atures, three-component neutral winds, and neutral ion species. Majeed et al. [2005] also used fractional joule heating (15\% efficiency) to provide energy balance while matching the temperature profile measured by the Galileo probe near the Jovian equator. Here, we use the same version of the JTGCM to reproduce existing auroral temperature data derived from various multispectral observations.

[46] The available temperature data for Jupiter's auroral thermosphere, both the northern and southern hemispheres, have been derived from a number of observations of bright emissions (either $\mathrm{H}_{2}$ or $\mathrm{H}_{3}^{+}$) emanating from different pressure regimes and from different Jovigraphical locations. Rather than comparing each temperature observation with the simulated temperature profile, we compare measured temperatures with an average temperature profile for the entire Jovian oval or polar cap regions. Figure 2 a shows such an average temperature profile for the northern (dashed curve) and southern (solid curve) oval regions in comparison with measured temperature data and a model temperature profile calculated by Grodent et al. [2001] using their 1-D electron transport model.

[47] The JTGCM temperatures at nanobar levels are found to be in reasonably good agreement with those inferred from the analysis of CFHT high-resolution mapping of the $\mathrm{H}_{3}^{+}$emissions from both the northern (green triangle and diamond) and southern (blue asterisk) oval regions [Raynaud et al., 2004b]. The temperatures derived from UKIRT near the $\mathrm{H}_{3}^{+}$peak $(\sim 0.1 \mu$ bar $)$ from both the northern and southern intense $\mathrm{H}_{3}^{+}$emissions (black asterisk and square, respectively) are also in good agreement with those simulated by the JTGCM. Note that the thermospheric temperature for the southern auroral region observed with both CFHT and UKIRT is warmer than the northern auroral region, consistent with the JTGCM simulation.

[48] The effective $\mathrm{H}_{2}$ temperatures derived from FUSE high-resolution UV spectra (from the northern Jovian aurora) represent three different pressure regimes in response to three spectral windows (920-970 ̊, 1030-1080 , and 1090$1180 \AA$ A) [Gustin et al., 2004a]. The FUSE temperature at $0.01 \mu$ bar derived from the first spectral window (920$970 \AA$ ) (red diamond) is very close to the JTGCM temperature, while the temperatures from the other two spectral windows (red triangle and red square) at around $1 \mu$ bar are approximately $400 \mathrm{~K}$ warmer than those simulated by the JTGCM. This discrepancy may reflect differences between the two approaches for data analysis adopted by Gustin et al. [2004a], a single-layer model that assumes a thin emitting layer with an effective energy for the $\mathrm{H}_{2}$ exciting electrons, and a multilayer model that assumes many vertically distributed energy degradation levels with a multiMaxwellian energy distribution function containing both hard $(>100 \mathrm{keV})$ and soft $(<1 \mathrm{keV})$ electron precipitation. Synthetic spectra have been used in both approaches to derive $\mathrm{H}_{2}$ gas temperatures and $\mathrm{H}_{2}$ self-absorbing column densities. Because the Gustin et al. model is a one-dimensional energy transport model with IR radiation (via $\mathrm{H}_{3}^{+}, \mathrm{CH}_{4}$, and $\mathrm{C}_{2} \mathrm{H}_{2}$ ) as the sole cooling mechanism, we suggest that the heating caused by precipitating electrons in this model is not being conducted downward rapidly enough below the homopause to radiate away by hydrocarbon cooling the excess warmth of the $\mathrm{H}_{2}$ responsible for the UV emissions at wavelengths longward of $1030 \AA$. Apparently, the Gustin et al. model was needed to fit the FUSE data; if they had lower temperatures in their model at $1 \mu$ bar, presumably they would get a poorer fit to the spectrum. Figure $2 \mathrm{a}$ also shows the derived temperature near the homopause from HST-STIS (purple square) observation of intense $\mathrm{H}_{2}$ emissions characterizing the main northern auroral oval. This temperature is in excellent agreement with the JTGCM temperature. A comparison between the HST-STIS (purple square) and FUSE (red triangle and red square) temperatures in the vicinity of $1 \mu \mathrm{bar}$ is also made. Clearly, a careful reanalysis of the FUSE data is needed.

[49] The vertical thermal structure of the Jovian stratosphere for pressure $>2 \mu$ bar has been observed with groundbased occultation of HIP9369 and Beta Scorpii by Jupiter's northern and southern polar regions, respectively. Figure $2 \mathrm{a}$ shows a comparison between observed temperature profiles and those simulated by the JTGCM. The simulated temperature profile in the north is in reasonably good agreement with those inferred from HIP9369 occultation data (both ingress and egress; light green curve) for pressure $>3 \mu \mathrm{bar}$. However, in the south, the JTGCM temperatures between 2 and $10 \mu \mathrm{bar}$ are found to be considerably larger than those derived from Beta Scorpii occultation data (both ingress and egress; red solid and red dotted curves). For this simulation, which incorporates $15 \%$ joule heating in the auroral oval, it appears that IR cooling processes are still competing with dynamical terms of heat transport to increase hydrocarbon radiation. Thus, the thermal budget in the Jovian southern stratosphere appears to be dominated and controlled by the resulting net heating from hydrodynamic advection and adiabatic processes. The simulated altitude profiles of such heating and cooling rates will be discussed in section 7 .

[50] Figure 2a also shows a direct comparison of JTGCM temperature profiles with the temperature profile calculated with a 1-D energy degradation model described by Grodent et al. [2001]. The main source of heating for the 1-D temperature profile is due to precipitated energetic electrons described by a three-component Maxwellian distribution. This distribution represents discrete auroral arcs (or ovals) [Grodent et al., 2001]. Since the JTGCM incorporates a vertical profile of auroral heating from this 1-D model to a three-dimensional grid and prescribes it around the main auroral ovals (see section 4 for details), this comparison provides critical information on the auroral thermal structures calculated from one- and three-dimensional approaches.

[51] Clearly, the temperature profile calculated with the $1-\mathrm{D}$ model is quite different from the one simulated by the JTGCM. The exospheric temperature from the 1-D model is about $300 \mathrm{~K}$ and $125 \mathrm{~K}$ larger than the exospheric temperature from the JTGCM for the northern and southern ovals, respectively. While the soft component of energy distribution (100 eV electrons) is primarily responsible for maintaining such a large exospheric temperature in both the 1-D model and the JTGCM, the difference in temperatures between the two models may be explained by examining heat transport processes. The 1-D model lacks heat transport processes related to the Jovian wind system. The heat transported to lower altitudes by thermal conduction alone in the 1-D model is not as efficient for excess heat removal as dynamical cooling [Achilleos et al., 1998; Bougher et al., 2005].

[52] The 1-D model also shows a quite different temperature structure between $1 \mu$ bar and 1 nbar compared to the JTGCM profiles. One possible reason for this difference is 
the Jovian wind system, which plays a significant role in redistributing thermospheric heat (see section 7 for details). Note that the temperatures from both the 1-D model and the JTGCM are in reasonably good agreement for the region $>1 \mu$ bar, since the heat in this region is primarily radiated away by hydrocarbon cooling (via $\mathrm{CH}_{4}$ and $\mathrm{C}_{2} \mathrm{H}_{2}$ emissions at 7.8 and $12.6 \mu \mathrm{m}$, respectively). Both models incorporate similar hydrocarbon cooling rates to balance heating rates below the homopause.

[53] Figure 2b shows the JTGCM temperature profiles averaged over the polar cap region for both hemispheres. Because of the differences in net heating caused by dynamical terms, the northern polar cap region appears to be much cooler than the southern polar cap region. A comparison between the polar cap and oval temperatures allows interesting insight into the thermal structure driven by very strong ion drag and joule heating processes that take place in the auroral thermospheres of the two Jovian hemispheres. The temperatures in the south are generally larger than those in the north perhaps because of similar power inputs from particle and joule heating into a smaller region in the south. The shapes of the profiles reflect the importance of various mechanisms that may control the thermal budget at different heights in the thermosphere. The exospheric temperatures of about 975 and $1175 \mathrm{~K}$ for the Jovian ovals in the north and south, respectively, are maintained largely by the balance between precipitated charged particle heating and adiabatic cooling caused by the upward flow of the neutral atmosphere. However, the polar cap temperatures of almost the same magnitudes in the north and south are maintained by different heating mechanisms. Joule heating is the dominant source of heating in the north even at $15 \%$ efficiency, while molecular conduction and joule heating in the south provide the necessary heating to control the thermal budget and to maintain the high magnitudes of exospheric temperatures.

[54] The distribution of oval and polar cap temperatures between 1 and $0.01 \mu$ bar seems to behave quite differently in the north and south. This region of the thermosphere is quite complicated and is primarily controlled by the Jovian dynamics, and ion drag forcing and joule heating contribute to a very strong thermospheric circulation of neutral winds (see Figure 1). These winds can play an important role in redistributing high-latitude heat toward the equator and throughout the Jovian thermosphere [e.g., Achilleos et al., 1998; Bougher et al., 2005; Majeed et al., 2005]. The horizontal neutral and vertical winds give rise to net heating and cooling of the thermosphere through hydrodynamic advection and adiabatic processes. The magnitude of net heating and cooling from these dynamical processes depends on which direction the wind is blowing. Thus the distribution of oval and polar cap temperatures in the north and south can be interpreted as because of north-south asymmetry in the circulation, which is linked to hemispheric differences in joule heating and the ion drifts themselves, as discussed in section 5. Using the argument of ion neutral coupling and simulated vertical profiles of heating and cooling rates (see section 7), one can explain the rapidly rising oval temperatures both in the north and south at around $0.2 \mu$ bar by the resulting net heating rate, which is primarily controlled by joule heating. However, the rise in polar cap temperatures in both hemispheres at around the same altitude cannot be interpreted by joule heating alone. The net heating in the southern polar cap is due to adiabatic compression of the neutral flow, while joule heating is the dominant source of net heating in the northern polar cap and maintains the neutral temperatures between 0.2 and $0.01 \mu$ bar. Note that the thermal structure of the lower thermosphere $(>1 \mu \mathrm{bar})$ simulated by the JTGCM for both the oval and polar cap is quite interesting. The temperature in the oval and polar cap decreases to $190 \mathrm{~K}$ from $1 \mu$ bar to the lower boundary of the JTGCM (20 $\mu$ bar $)$ in both hemispheres. However, cooling in the south appears to be much slower than in the north, resulting in much larger temperatures in the southern thermosphere than in the northern thermosphere (see Figures $2 \mathrm{a}$ and $2 \mathrm{~b}$ ). Since the source of heating in this part of the thermosphere is due to dynamical terms (adiabatic and hydrodynamic advection processes), the radiative cooling of this transported heat still needs to be balanced. This also suggests that steady state conditions in JTGCM have not been achieved for pressures $>1 \mu \mathrm{bar}$.

[55] Finally, the only available polar cap temperature derived from HST-STIS observation of Jupiter's northern aurora is compared with the JTGCM temperature profile simulated for the northern polar cap region. Figure $2 \mathrm{~b}$ shows that the measured temperature near the Jovian homopause is in excellent agreement with the JTGCM temperature.

\section{Auroral Thermal Balance}

[56] We have calculated the vertical profiles of thermal balances associated with each source from the JTGCM simulation that best describes the measured temperatures from multispectral observations of the Jovian aurora. The balance between heating and cooling terms averaged over the Jovian oval and polar cap regions is shown in Figures 3 and 4 , respectively.

\subsection{Southern Auroral Oval Region}

[57] Figure 3a illustrates the balance between auroral heating caused by precipitating charged particles and corresponding adiabatic cooling caused by neutral outflows to maintain an exospheric temperature of about $1180 \mathrm{~K}$ in the southern oval, consistent with the CFHT temperature derived from $2-\mu \mathrm{m}$ auroral emissions. Joule heating appears to play a minor role in heating the upper atmosphere. Molecular conduction provides a comparable cooling for pressures $<0.1$ nbar and becomes a minor source of heating for the region between 1 and 0.2 nbar. In the thermosphere between $1 \mu$ bar and 1 nbar, the Jovian wind system seems to play an active role in transporting energy from the southern oval to the rest of the planet. Figure $3 \mathrm{~b}$ shows that joule heating and forcing by ion drag dominate the heat budget, with a peak value of $\sim 3.7 \times 10^{7} \mathrm{eV} \mathrm{cm}^{-3} \mathrm{~s}^{-1}$

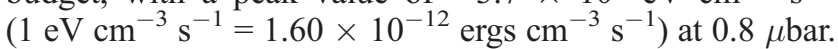
This tremendous amount of heat is balanced by cooling caused by horizontal advection, induced by meridional flow with a maximum velocity of $\sim-0.1 \mathrm{~km} \mathrm{~s}^{-1}$ [e.g., Bougher et al., 2005]. This thermal balance yields a temperature of about $500 \mathrm{~K}$ at $0.8 \mu$ bar. A rapid increase in oval temperature is seen between 0.3 and $0.02 \mu$ bar (see Figure 2a) owing to the net heating rate, which appears to be controlled more by the ion drag process compared to other sources. 

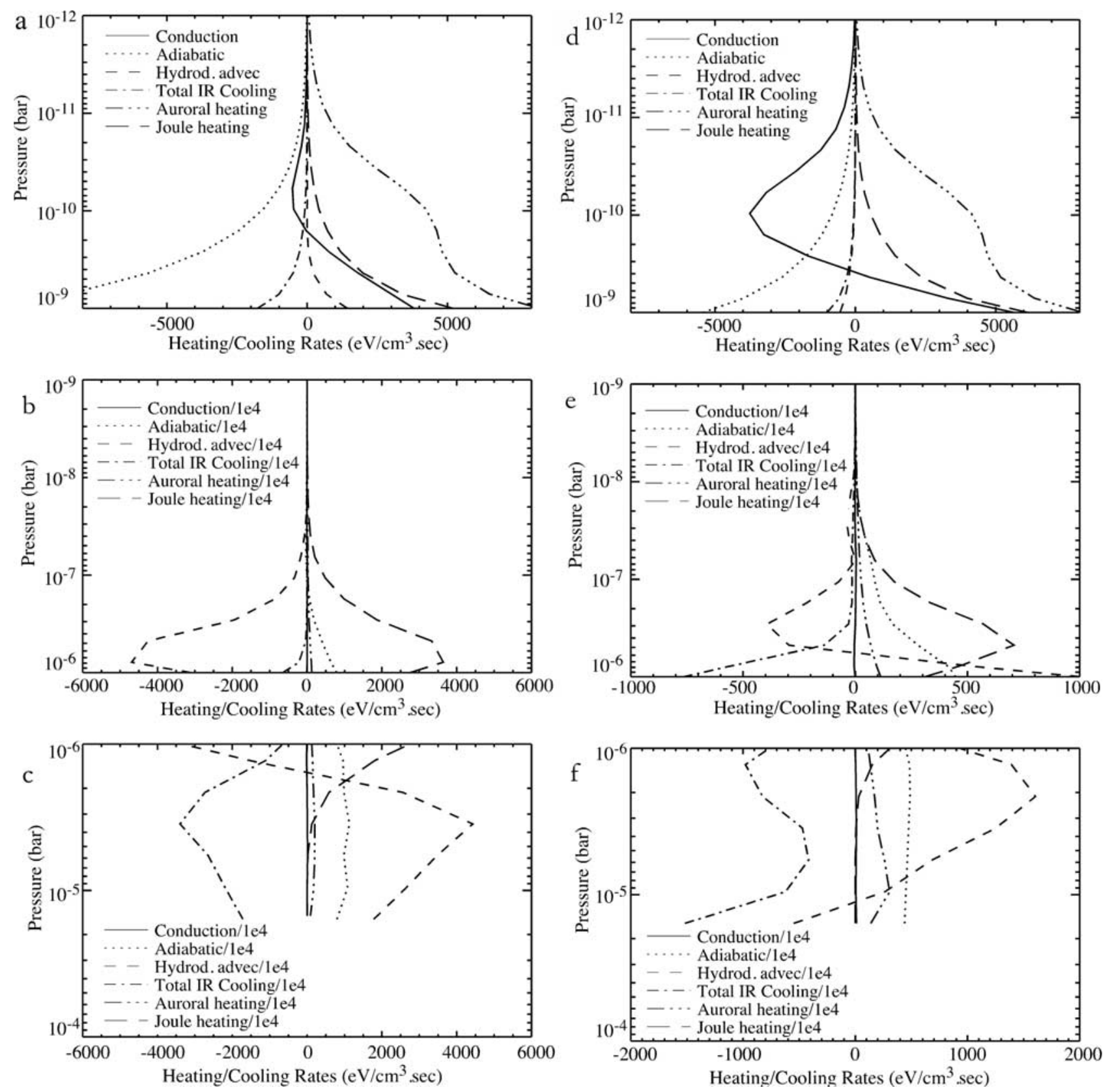

Figure 3. $(\mathrm{a}-\mathrm{c})$ Average altitude profiles of heating and cooling rates are shown for the JTGCM simulation of the southern auroral oval. Three pressure regions are shown, $10^{-9}$ to $10^{-12}$ bar (Figure 3a), $10^{-6}$ to $10^{-9}$ bar (Figure $3 \mathrm{~b}$ ), and $10^{-4}$ to $10^{-6}$ bar (Figure $3 \mathrm{c}$ ). Note that the heating and cooling rates are scaled. $(\mathrm{d}-\mathrm{f})$ Same as Figures $3 \mathrm{a}-3 \mathrm{c}$ but for northern auroral oval.

[58] Note that the Jovian upper atmosphere from the homopause $(\sim 1 \mu$ bar $)$ to the lower boundary is the most complex region of the JTGCM. The competing processes responsible for controlling the thermal structure have not yet reached equilibrium. Downward motion is still causing atmospheric compression while meridional flow is still transporting heat away from the southern oval. The net heating rate for the thermal structure in this region is determined by the competition between heat transport processes and radiative cooling processes. Figure $3 \mathrm{c}$ reveals the importance of vertical energy transport by dynamical terms below the homopause $(>1 \mu \mathrm{bar})$. The transport processes associated with downwelling winds and meridional flow appear to generate enough heat, with a maximum value of $\sim 5.5 \times$ $10^{7} \mathrm{eV} \mathrm{cm}^{-3} \mathrm{~s}^{-1}$ near $3.5 \mu \mathrm{bar}$, to be balanced with hydrocarbon cooling resulting from $\mathrm{CH}_{4}(7.8 \mu \mathrm{m})$ and $\mathrm{C}_{2} \mathrm{H}_{2}(13.4 \mu \mathrm{m}$ ) radiation (with a maximum value of $3.5 \times$ $10^{7} \mathrm{eV} \mathrm{cm}^{-3} \mathrm{~s}^{-1}$ at $\sim 0.3 \mu$ bar). The effect of such an imbalance on the simulated thermal structure can be seen in rising temperatures from the lower boundary to about $2 \mu$ bar compared to those inferred from Beta Scorpii occultation (egress and ingress) of the southern aurora (see Figure 2a). Note that dynamical cooling is suddenly increased between 1 and $2 \mu \mathrm{bar}$, causing a "bump" in the JTGCM temperature, as shown in Figure 2a. 

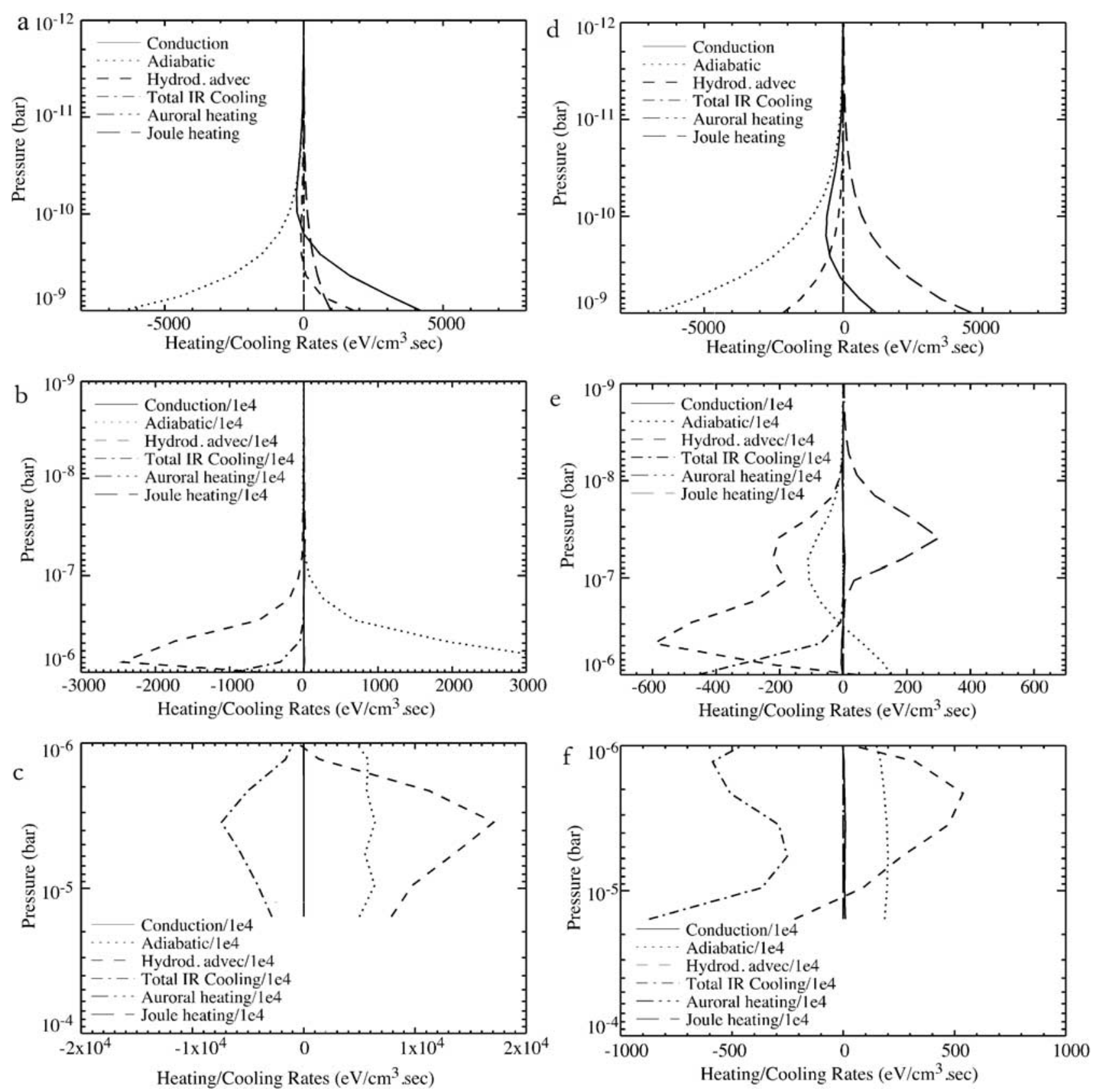

Figure 4. $(\mathrm{a}-\mathrm{c})$ Average altitude profiles of heating and cooling rates are shown for the JTGCM simulation of the region poleward of the southern auroral oval. Three pressure regions are shown, $10^{-9}$ to $10^{-12}$ bar (Figure $4 \mathrm{a}$ ), $10^{-6}$ to $10^{-9}$ bar (Figure $4 \mathrm{~b}$ ), and $10^{-4}$ to $10^{-6}$ bar (Figure $4 \mathrm{c}$ ). Note that the heating and cooling rates are scaled. $(\mathrm{d}-\mathrm{f})$ Same as Figures $4 \mathrm{a}-4 \mathrm{c}$ but for the region poleward of the northern auroral oval.

\subsection{Northern Auroral Oval Region}

[59] Figures $3 \mathrm{~d}-3 \mathrm{f}$ show the average heating and cooling rates that best describe the Jovian thermal structure confined to the northern oval region. Note that charged particle heating is unchanged from the southern oval because of its parameterization within the JTGCM to prescribe identical heating rate profiles around the Jovian ovals in both hemispheres. Figure $3 \mathrm{~d}$ illustrates that such an intense particle heating in the northern oval is primarily balanced with cooling generated by molecular conduction between $0.3 \mathrm{nbar}$ and the altitude of the top boundary in the JTGCM at around
$10^{-4}$ nbar. Charged particle heating continues to dominate from 0.3 nbar to 1 nbar with minor contributions from joule heating and conduction processes. This heating is largely offset by cooling provided by adiabatic expansion of the neutral atmosphere due to upwelling. These balances appear to maintain an exospheric temperature of about $980 \mathrm{~K}$ (see Figure 2a), consistent with those derived from CFHT observations of the Jovian northern aurora.

[60] As discussed above, the estimated ion drifts in the northern oval are smaller than those in the southern oval, owing to the mapping of VIP4 magnetic field lines [e.g., 
Connerney et al., 1998]. This results in smaller ion drag forcing and corresponding joule heating in the northern oval region compared to that in the southern oval region [Bougher et al., 2005]. Figure 3e shows the impact of this reduced joule heating on the underlying thermosphere of the northern oval region. Although smaller in magnitude, joule heating seems to serve as a dominant source of thermal budget between $1 \mu \mathrm{bar}$ and $0.3 \mathrm{nbar}$. As it tends to heat the thermosphere with a maximum heating rate of $\sim 0.7 \times$ $10^{7} \mathrm{eV} \mathrm{cm}^{-3} \mathrm{~s}^{-1}$ at around $0.5 \mu \mathrm{bar}$, horizontal advection induced by meridional flow and IR radiation tends to provide the necessary cooling with a maximum rate of $\sim 0.4 \times$ $10^{7} \mathrm{eV} \mathrm{cm}^{-3} \mathrm{~s}^{-1}$ and $\sim 0.7 \times 10^{7} \mathrm{eV} \mathrm{cm}^{-3} \mathrm{~s}^{-1}$ at $0.3 \mu \mathrm{bar}$ and $1 \mu$ bar, respectively. It is important to note that the net thermal budget is quite well balanced between 1 and $0.2 \mu$ bar, so the resulting thermospheric temperature behaves accordingly. However, as the cooling decreases below $0.2 \mu \mathrm{bar}$, ion drag forcing (or joule heating) becomes the dominant source of net heating, which apparently serves to raise oval temperature rapidly from 0.2 to $0.01 \mu$ bar (see Figure 2a). The thermal balance for the lower thermosphere $(>1 \mu$ bar) in the northern oval region seems to be largely determined by competition between dynamical heat and corresponding hydrocarbon cooling. Figure $3 \mathrm{f}$ shows that strong dynamical heating, mainly due to the horizontal advection process with a minor contribution from adiabatic compression of neutral flow, is in control of the net thermal budget of the lower thermosphere, with a peak value of $\sim 2 \times 10^{7} \mathrm{eV} \mathrm{cm}^{-3} \mathrm{~s}^{-1}$ at $\sim 2 \mu$ bar. This strong dynamical heating in the JTGCM is regulated by an extremely large amount of cooling resulting from $\mathrm{CH}_{4}(7.8 \mu \mathrm{m})$ and $\mathrm{C}_{2} \mathrm{H}_{2}$ $(12.6 \mu \mathrm{m})$ radiation. The net impact of this thermal balance on the Jovian thermosphere can be seen from the distribution of neutral temperature, as shown in Figure $2 \mathrm{a}$.

\subsection{Southern Polar Cap Region}

[61] As discussed above, the Jovian polar caps have been investigated with observations of UV emissions [Grodent et al., 2003; Gustin et al., 2002] in addition to some "flare-like" emissions [Waite et al., 2001], and X-ray emissions [Gladstone et al., 2002; Branduardi-Raymont et al., 2007, 2008]. Compared to the main oval emission, polar cap emissions map along magnetic field lines further out in the magnetosphere, at radial distances greater than $30 R j$, or perhaps to open field lines connecting to the interplanetary magnetic field [e.g., Cowley et al., 2005]. The corresponding ions are subject to corotation breakdown and thereby influence the corotating neutrals in unique ways. Some of the magnitudes of ion drifts, specifically near the Jovian poles (estimated by Bougher et al. [2005]), are comparable to those of the main oval regions. These drifts can effectively modify the neutral winds in the Jovian polar caps, thereby affecting the vertical distribution of the thermal budget. Figure 4 shows vertical profiles of heating and cooling rates averaged over the southern and northern polar regions.

[62] Figure 4a shows vertical profiles of heating and cooling rates for the southern polar cap region between 1 and 0.001 nbar from various competing processes. Clearly, the total heating is controlled by thermal conduction between 1 and 0.1 nbar with an additional contribution from ion drag (or joule heating). The bulk of this heating is largely balanced by adiabatic cooling, to maintain an exospheric temperature of about $1200 \mathrm{~K}$. Note that the Jovian wind system is quite active in transporting heat from 1 nbar to the lower boundary of the JTGCM. Figure 4b shows the competition between adiabatic heating and hydrodynamic cooling between $1 \mu \mathrm{bar}$ and 1 nbar. The maximum heating rate from the adiabatic process is $\sim 3.5 \times 10^{7} \mathrm{eV} \mathrm{cm}^{-3} \mathrm{~s}^{-1}$ at $0.8 \mu \mathrm{bar}$, whereas the maximum cooling from the hydrodynamic advection process at the same pressure is $22.5 \times 10^{7} \mathrm{eV} \mathrm{cm}^{-3} \mathrm{~s}^{-1}$. The resulting net heating in this region appears to cause the thermospheric temperature to rise from $\sim 750 \mathrm{~K}$ at $1 \mu \mathrm{bar}$ to $\sim 1100 \mathrm{~K}$ at $0.1 \mu \mathrm{bar}$ (see Figure $2 \mathrm{~b}$ ). The dynamical heat processes continue to dominate the thermal budget from $1 \mu \mathrm{bar}$ to the lower boundary of the JTGCM. As can be seen in Figure 4f, some of the combined heat from horizontal advection and adiabatic compression processes is radiated away by IR radiation, while the excess heat apparently serves to raise the thermospheric temperature from the lower boundary up to $2 \mu$ bar (see Figure $2 b$ ).

\subsection{Northern Polar Cap Region}

[63] The energy situation in the northern polar cap is different owing to smaller simulated magnitudes of the neutral horizontal winds compared to the southern polar cap. Figures $4 \mathrm{~d}-4 \mathrm{f}$ show the average vertical profiles of heating and cooling rates for the northern polar cap region, covering the entire thermosphere simulated by the JTGCM. Note that the main source of heat in the upper region of the thermosphere is dominated by ion drag (or joule heating). The corresponding cooling is provided by heat transport (Figure 4d) with the major contribution from adiabatic expansion resulting from outward neutral flow. Figure $4 \mathrm{e}$ shows that dynamical cooling, mainly from hydrodynamic advection, continues to dominate the thermal budget in the Jovian thermosphere from $1 \mu$ bar to 1 nbar, with a maximum cooling rate of $\sim 0.6 \times 10^{7} \mathrm{eV} \mathrm{cm}^{-3} \mathrm{~s}^{-1}$ at about $0.5 \mu \mathrm{bar}$. However, the heat in this region of the thermosphere is primarily provided by joule heating somewhat higher in the thermosphere, with a maximum heating rate of $\sim 0.3 \times$ $10^{7} \mathrm{eV} \mathrm{cm}^{-3} \mathrm{~s}^{-1}$ at about $0.04 \mu$ bar. Although these simulated magnitudes of heating and cooling are smaller than those for the southern polar cap region, the wind transport processes seem to yield a temperature profile (see Figure $2 \mathrm{~b}$ ) consistent with thermal balances between 1 nbar and $1 \mu$ bar. Figure 4f illustrates the thermal balance for the lowest part of the Jovian thermosphere ( $>1 \mu$ bar). The local dynamical heating is mainly due to the horizontal advection process and reaches a maximum value of $\sim 0.8 \times 10^{7} \mathrm{eV} \mathrm{cm}^{-3} \mathrm{~s}^{-1}$ at about $2 \mu$ bar, more than an order of magnitude smaller than that simulated for the southern polar cap region. An effective sink to this heat is provided by an extremely large amount of IR cooling resulting from $\mathrm{CH}_{4}(7.8 \mu \mathrm{m})$ and $\mathrm{C}_{2} \mathrm{H}_{2}(12.6 \mu \mathrm{m})$ radiation. This IR cooling in the JTGCM is sufficiently large between 1 and $20 \mu$ bar to maintain an isothermal layer of $\sim 190 \mathrm{~K}$.

\section{Integrated Auroral Thermal Balances}

[64] Total heating simulated by the JTGCM, both in the oval and polar cap regions, should be reflected in the corresponding total cooling that results from its dissipation. Tables $2 \mathrm{a}$ and $2 \mathrm{~b}$ summarize the calculated column-integrated heating and cooling rates from the thermal processes 
Table 2a. Average Column Integrated Thermal Balances for the South and North Polar Cap Region ${ }^{\mathrm{a}}$

\begin{tabular}{lcccc}
\hline & \multicolumn{3}{c}{ Pressure } \\
\cline { 2 - 5 } \multicolumn{1}{c}{ Process } & $20 \mu$ bar to $1.4 \mathrm{nbar}$ & $<1 \mu$ bar & $>1 \mu$ bar & $<1 \mathrm{nbar}$ \\
\hline Conduction & $1.31(-0.52)$ & $-1.2(-0.05)$ & $0.36(0.48)$ & $0.08(-0.03)$ \\
Adiabatic & $650.5(-12.0)$ & $265.1(28.5)$ & $399.5(12.8)$ & $-0.18(-0.25)$ \\
Advection & $-132.9(-133.4)$ & $31.9(-71.86)$ & $844.6(17.4)$ & $0.015(-0.06)$ \\
Total IR cooling & $-125.8(-66.9)$ & $-67.6(-23.5)$ & $-360.0(-24.2)$ & $-1.2 \times 10^{-8}\left(-9.9 \times 10^{-9}\right)$ \\
Particle heating & $0(0)$ & $0(0)$ & $0(0)$ & $0(0)$ \\
Joule heating & $4.8(86.4)$ & $4.5(80.5)$ & $3.6 \times 10^{-6}\left(9.0 \times 10^{-6}\right)$ & $0.03(0.16)$ \\
Total heating & $656.6(86.4)$ & $681.5(109.0)$ & $1244.5(30.6)$ & $0.125(0.16)$ \\
Total cooling & $258.7(212.8)$ & $68.8(95.4)$ & $360.0(24.2)$ & $0.18(0.34)$ \\
\hline
\end{tabular}

${ }^{\mathrm{a}}$ Units are erg cm${ }^{-2} \mathrm{~s}^{-1}\left(1 \mathrm{erg} \mathrm{cm}^{-2} \mathrm{~s}^{-1}=1 \mathrm{~mW} \mathrm{~m}^{-2}\right.$ for those who prefer the SI units). Values not in parenthesis are for the southern hemisphere and values in parenthesis are for the northern hemisphere.

(see Figures 3 and 4) that compete to control the bulk of the thermal budget and to maintain thermospheric temperatures consistent with those derived from multispectral observations of different regions of Jupiter's aurora. Note that these rates are calculated and represented in ergs $\mathrm{cm}^{-2} \mathrm{~s}^{-1}\left(1 \mathrm{erg} \mathrm{cm} \mathrm{cm}^{-2} \mathrm{~s}^{-1}\right.$ is equal to $1 \mathrm{mWm}^{-2}$ for those who prefer SI units).

[65] The column-integrated heating rates for the entire thermosphere from 1.4 nbar to $20 \mu$ bar are generally more intense compared to the column-integrated cooling rates in both hemispheres (see Tables $2 \mathrm{a}$ and $2 \mathrm{~b}$ ). In general, joule heating appears to be the main contributor to this intense integrated heating both in the oval and the polar cap regions. This reflects the enhanced role of the ion drag process, which is responsible for intensifying neutral winds in the Jovian high-latitude region, thereby affecting the distribution of neutral temperature. On the contrary, the column-integrated cooling of the Jovian oval and polar cap regions are determined by different processes. For the southern high-latitude region, the horizontal advection process dominates the Jovian cooling both in the oval $(\sim 90 \%)$ and polar cap $(\sim 55 \%)$ regions along with a contribution from hydrocarbon radiation mainly from $\mathrm{CH}_{4}$ emission at $7.8 \mu \mathrm{m}$ [cf. Yelle et al., 2001]. However, in the northern oval region, this IR cooling becomes the main contributor (almost 100\%) to the columnintegrated cooling rate. The column-integrated cooling rate in the northern polar cap is controlled by a combination of hydrocarbon cooling from IR radiation $(\sim 33 \%)$ and dynamical cooling $(\sim 66 \%)$ mainly from the horizontal advection process. The bulk of this strong IR cooling and dynamical cooling serves to balance the large amount of heat produced in the Jovian oval and polar cap regions.
[66] Clearly, for pressure $>1 \mu$ bar, hydrodynamic advection plus adiabatic compression of the neutral flow dominates the heat budget (1244.5 and 306.4 ergs $\mathrm{cm}^{-2} \mathrm{~s}^{-1}$ for the southern polar cap and oval regions, respectively, compared to 30.6 and $86.8 \mathrm{ergs} \mathrm{cm}^{-2} \mathrm{~s}^{-1}$ for the northern polar cap and oval regions, respectively) while hydrocarbon

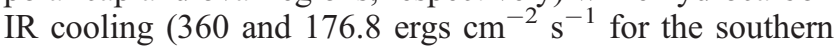
polar cap and oval regions, respectively, compared to 24.2 and 30.1 ergs $\mathrm{cm}^{-2} \mathrm{~s}^{-1}$ for the northern polar cap and oval regions, respectively) tends to radiate most of this heat away from both the oval and polar cap regions of the southern and northern hemispheres. The resulting net heating of about 884 and 130 ergs $\mathrm{cm}^{-2} \mathrm{~s}^{-1}$ for the southern polar cap and oval, respectively, can be used to interpret much warmer temperatures from the lower boundary of the JTGCM to $2 \mu$ bar (see Figures $2 \mathrm{a}$ and $2 \mathrm{~b}$ ). It is important to note that the simulated temperature in this region of the thermosphere $(>1 \mu$ bar) does not yet approach steady state conditions even at the end of 84 Jovian days of model integration time.

[67] Tables $2 \mathrm{a}$ and $2 \mathrm{~b}$ also show the average columnintegrated heating and cooling rates for the upper thermospheric regions $(<1 \mu$ bar and $<1$ nbar $)$ for which the steady state conditions for temperature and neutral wind fields prevail. The column-integrated heating rate in the southern oval region for pressure less than $1 \mu$ bar resulting from joule heating and particle heating reaches a maximum value of $289.1 \mathrm{ergs} \mathrm{cm} \mathrm{cm}^{-2} \mathrm{~s}^{-1}$. This value is reduced to about $0.74 \mathrm{ergs} \mathrm{cm}^{-2} \mathrm{~s}^{-1}$ for the exospheric region for pressure $<1$ nbar. These heating rates are mostly offset by cooling because of horizontal advection for pressure $<1 \mu$ bar and by the adiabatic process for pressure $<1$ nbar. Note that integrated heating rates in the southern polar cap region are different than those in the southern oval region because of

Table 2b. Average Column Integrated Thermal Balances for the South and North Auroral Oval Region ${ }^{\mathrm{a}}$

\begin{tabular}{lcccc}
\hline & \multicolumn{3}{c}{ Pressure } \\
\cline { 2 - 5 } \multicolumn{1}{c}{ Process } & $20 \mu$ bar to 1.4 nbar & $<1 \mu$ bar & $>1 \mu$ bar & $<.29(0.49)$ \\
Conduction & $0.88(-0.52)$ & $-0.81(-0.03)$ & $69.3(30.4)$ & $0.07(-0.15)$ \\
Adiabatic & $100.4(94.6)$ & $34.5(49.0)$ & $218.5(39.7)$ & $-0.38(-0.17)$ \\
Advection & $-544.3(2.76)$ & $-189.3(-26.7)$ & $-176.8(-30.1)$ & $0.021(-0.01)$ \\
Total IR cooling & $-78.6(-91.2)$ & $-48.12(-34.6)$ & $11.9(15.6)$ & $-0.04(-0.02)$ \\
Particle heating & $28.9(27.5)$ & $18.2(17.6)$ & $6.4(0.6)$ & $0.51(0.5)$ \\
Joule heating & $661.5(233.3)$ & $236.4(130.9)$ & $306.4(86.8)$ & $0.14(0.17)$ \\
Total heating & $791.7(358.2)$ & $289.1(197.5)$ & $176.8(30.1)$ & $0.74(0.67)$ \\
Total cooling & $622.9(91.7)$ & $238.2(61.33)$ & $0.42(0.35)$ \\
\hline
\end{tabular}

${ }^{a}$ Units are erg cm ${ }^{-2} \mathrm{~s}^{-1}\left(1 \mathrm{erg} \mathrm{cm}^{-2} \mathrm{~s}^{-1}=1 \mathrm{~mW} \mathrm{~m}^{-2}\right.$ for those who prefer the SI units). Values not in parenthesis are for the southern hemisphere and values in parenthesis are for the northern hemisphere. 
the role of different thermal processes. The main contributor to the maximum heating rate $\left(\sim 301.5 \mathrm{ergs} \mathrm{cm}^{-2} \mathrm{~s}^{-1}\right)$ in the southern polar cap region is the adiabatic process for pressure $<1 \mu \mathrm{bar}$, while for this region downward conduction plus joule heating $\left(\sim 0.11 \mathrm{ergs} \mathrm{cm} \mathrm{cm}^{-2} \mathrm{~s}^{-1}\right)$ dominates the peak heating rate for pressure $<1$ nbar. While the IR cooling process appears to dominate other processes for pressure $<1 \mu$ bar $\left(67.6 \mathrm{erg} \mathrm{cm}^{-2} \mathrm{~s}^{-1}\right)$ the dynamical cooling resulting from the adiabatic process yields the main source of cooling for pressure $<1 \mathrm{nbar}\left(0.18 \mathrm{ergs} \mathrm{cm}^{-2} \mathrm{~s}^{-1}\right)$ in the southern polar cap region.

[68] The average column-integrated heating and cooling rates in the northern oval and polar cap regions for pressures $<1 \mu$ bar and $<1$ nbar are also shown in Tables $2 \mathrm{a}$ and $2 \mathrm{~b}$. These rates are quite different than those for the southern auroral region. It is important to note that joule heating, although considerably smaller than the southern value, plays an important role in determining the neutral temperature distribution in the northern oval region. A combination of joule heating $(\sim 75 \%)$ and adiabatic heating $(\sim 25 \%)$ provides the total column-integrated heating rate for the northern polar cap region for pressure $<1 \mu$ bar. However, joule heating appears to control (with $\sim 100 \%$ contribution) the total column-integrated heating rate for this region for pressure $<1$ nbar. On the other hand, for the northern oval region, the total integrated heating rate for pressure $<1 \mu \mathrm{bar}$ is dominated by joule heating (130.9 $\mathrm{erg} \mathrm{cm}^{-2} \mathrm{~s}^{-1}$ ), while precipitation of charged particles provides almost $80 \%$ of the total integrated heating rate for the Jovian exosphere $(<1$ nbar). The necessary column-integrated cooling rate to dissipate such a strong column of heat in both the northern oval and polar cap regions is provided by wind transport and IR cooling processes. The cooling due to horizontal advection of the neutral winds seems to play an important role in providing the main source $(\sim 70 \%)$ of columnintegrated cooling rate with somewhat less contribution from the IR cooling process $(\sim 30 \%)$ in the northern polar cap for pressures $<1 \mu$ bar. In the northern oval, however, this combination splits into almost $50 \%$ to the total columnintegrated cooling rate. For pressures $<1$ nbar, the columnintegrated cooling in both the northern oval and polar cap regions is determined by the competition between the adiabatic process associated with neutral outflow and downward conduction. These processes contribute equally to the total integrated cooling in the northern oval region. However, adiabatic cooling provides almost $80 \%$ of the total integrated cooling in the northern polar cap region.

\section{Summary}

[69] A fully three-dimensional Jupiter Thermospheric General Circulation Model (JTGCM) is used to simulate the global dynamical structure of the Jovian thermosphere self-consistently with thermal structure and composition distributions from 250 to $3000 \mathrm{~km}$. This simulation is used to interpret available multispectral temperature data obtained for Jupiter's main auroral ovals and polar caps with groundbased instruments and Earth orbiting telescopes. The JTGCM has been integrated for 84 Jovian days to investigate the underlying thermal processes that maintain and drive enormous variations in the temperature structure. We have shown that the underlying global circulation of the neutral wind system, driven by moderate auroral heating and $15 \%$ of the total joule heating produced in the auroral ovals, can redistribute high-latitude heat on a global scale. It is also shown that cooling of the auroral regions is caused by strong outflows that develop near the ovals as a result of large-scale pressure gradients and magnetospheric forcing imposed by high-latitude ion convection. The pole-to-equator circulation of the neutral flow resulting from strong Coriolis torques acting on the equatorward-directed meridional wind, rising motion in the auroral ovals, and subsequent convergence and downwelling motion at the Jovian equator, can regulate the transport of energy outward from the auroral regions to the rest of the planet. We find that the average thermal budget for the Jovian ovals and polar caps is controlled by such circulation in a manner consistent with temperature observations on Jupiter. The main source of the upper thermospheric $(<1 \mu$ bar) column integrated heating in the Jovian ovals (both in the north and south) is due to joule heating. Various processes compete to provide adequate upper thermospheric $(<1 \mu$ bar $)$ column integrated heating in the Jovian polar caps because the auroral process of particle precipitation does not contribute to the total heating. We find that adiabatic heating, caused by atmospheric compression of the neutral flow, is an important process for the southern thermosphere between 1 and $0.01 \mu$ bar while heating due to thermal conduction and the joule heating processes dominates the exospheric region $(<1$ nbar). However, in the northern polar cap, joule heating primarily provides the most heating to the upper thermosphere $(<1 \mu$ bar) compared to other sources. Cooling of the upper thermosphere for both the ovals and polar caps is mainly controlled by wind transport processes such as adiabatic and hydrodynamic advection with an additional contribution from the IR cooling process near $1 \mu \mathrm{bar}$. The thermal budget for the lower thermosphere ( $>1 \mu$ bar; below the homopause) in both the ovals and polar caps is dominated by wind transport processes, and IR radiation mainly arises from $\mathrm{CH}_{4}$ and $\mathrm{C}_{2} \mathrm{H}_{2}$ emissions at 7.8 and $12.6 \mu \mathrm{m}$, respectively. We find that dynamical heat below the homopause is not efficiently radiated away via hydrocarbon emission from both the ovals and polar caps. This results in a net atmospheric imbalance that seems to provide an explanation for a rapid increase of the neutral temperature for the region with pressure greater than $2 \mu \mathrm{bar}$.

[70] Acknowledgments. We are grateful to the National Center for Atmospheric Research (NCAR) for the use of the IBM/SP and SGI supercomputer resources necessary to develop and exercise the JTGCM thermospheric model and its postprocessor. In addition, special thanks go to Ben Foster of NCAR for his help preparing the JTGCM code to run on the IBM/SP computers and to D. K. Eddy and A. Corelli for editorial support. The reviewer's comments on our work helped to improve the original manuscript and so are also highly acknowledged. This work is supported by NASA/STScI grant HST-AR-09941.01-A, NASA/Planetary Atmosphere grant NNG0SGB40G, and NSF grant AST-0406881 through subcontract 499951Q from the Southwest Research Institute to the University of Michigan.

\section{References}

Achilleos, N., S. Miller, J. Tennyson, A. D. Aylward, I. Mueller-Wodarg, and D. Rees (1998), JIM: A time-dependent, three-dimensional model of Jupiter's thermosphere and ionosphere, J. Geophys. Res., 103, 20,089-20,112, doi:10.1029/98JE00947.

Ajello, J. M., et al. (1998), Galileo orbiter ultraviolet observations of Jupiter aurora, J. Geophys. Res., 103, 20,125-20,148, doi:10.1029/98JE00832. Ajello, J. M., D. E. Shemansky, W. R. Pryor, A. I. Stewart, K. E. Simmons, T. Majeed, J. H. Waite, G. R. Gladstone, and D. Grodent (2001), Spectro- 
scopic evidence for high altitude aurora at Jupiter from Galileo extreme ultraviolet spectrometer and Hopkins ultraviolet telescope observations, Icarus, 152, 151-171, doi:10.1006/icar.2001.6619.

Ajello, J. M., W. Pryor, L. Esposito, I. Stewart, W. McClintock, J. Gustin, D. Grodent, G.-C. Gérard, and J. T. Clarke (2005), The Cassini Campaign observations of the Jupiter aurora by the Ultraviolet Imaging Spectrograph and the Space Telescope Imaging Spectrograph, Icarus, 178 327-345, doi:10.1016/j.icarus.2005.01.023.

Ballester, G. E., et al. (1996), Time-resolved observations of Jupiter's farultraviolet aurora, Science, 274, 409-412, doi:10.1126/science. 274.5286.409.

Baron, R. L., T. Owen, J. E. P. Connerney, T. Satoh, and J. Harrington (1996), Solar wind control of Jupiter's $\mathrm{H}_{3}^{+}$auroras, Icarus, 120, 437-442, doi:10.1006/icar.1996.0063.

Bhardwaj, A., et al. (2007), X-rays from solar system objects, Planet. Space Sci., 55, 1135-1189, doi:10.1016/j.pss.2006.11.009.

Bougher, S. W., S. Engel, R. G. Roble, and B. Foster (1999), Comparative terrestrial planet thermospheres: 2 . Solar cycle variation of global structure and winds at equinox, J. Geophys. Res., 104, 16,591-16,611, doi:10.1029/1998JE001019.

Bougher, S. W., J. H. Waite, T. Majeed, and G. R. Gladstone (2005), Jupiter Thermosphere General Circulation Model (JTGCM): Global studies and dynamics driven by auroral and joule heating, J. Geophys. Res., 110, E04008, doi:10.1029/2003JE002230.

Branduardi-Raymont, G. A., et al. (2007), A study of Jupiter's aurorae with XMM-Newton, Astron. Astrophys., 463, 761-774, doi:10.1051/ 0004-6361:20066406

Branduardi-Raymont, G. A., et al. (2008), Spectral morphology of the X-ray emission from Jupiter's aurorae, J. Geophys. Res., 113, A02202, doi:10.1029/2007JA012600.

Broadfoot, A. L., et al. (1979), Extreme ultraviolet observations from Voyager 1 encounter with Jupiter, Science, 204, 979-982, doi:10.1126/ science. 204.4396 .979 .

Bunce, E. J., and S. W. H. Cowley (2001), Divergence of the equatorial current in the dawn sector of Jupiter's magnetosphere: Analysis of Pioneer and Voyager magnetic field data, Planet. Space Sci., 49, 1089-1113, doi:10.1016/S0032-0633(01)00004-6.

Caldwell, J., B. Turgeon, and X.-M. Hua (1992), Hubble Space Telescope imaging of the north polar aurora on Jupiter, Science, 257, 1512-1515, doi:10.1126/science.257.5076.1512.

Carbary, J. F., S. M. Krimigis, E. P. Keath, G. Gloeckler, W. I. Axford, and T. P. Armstrong (1981), Ion anisotropies in the outer Jovian magnetosphere, J. Geophys. Res., 86, 8285-8299, doi:10.1029/ JA086iA10p08285.

Clarke, J. T., L. Ben Jaffel, A. Vidal-Madjar, G. R. Gladstone, J. H. Waite Jr., R. Prange, J.-C. Gerard, J. Ajello, and G. James (1994), Hubble Space Telescope Goddard high-resolution spectrograph $\mathrm{H}_{2}$ rotational spectra of Jupiter's aurora, Astrophys. J., 430, L73-L76, doi:10.1086/187441.

Clarke, J. T., et al. (1996), Far-UV imaging of Jupiter's aurora with HST/ WFPC 2, Science, 274, 404-409, doi:10.1126/science.274.5286.404.

Clarke, J. T., et al. (1998), Hubble Space Telescope imaging of Jupiter's UV aurora during the Galileo orbiter mission, J. Geophys. Res., 103, 20,217-20,236, doi:10.1029/98JE01130.

Connerney, J. E. P., R. Baron, T. Satoh, and T. Owen (1993), Images of excited $\mathrm{H}_{3}^{+}$at the foot of the Io flux tube in Jupiter's atmosphere, Science, 262, 1035-1038, doi:10.1126/science.262.5136.1035.

Connerney, J. E. P., M. H. Acuna, N. F. Ness, and T. Satoh (1998), New models of Jupiter's magnetic fields constrained by the Io flux tube footprint, J. Geophys. Res., 103, 11,929-11,940, doi:10.1029/97JA03726.

Cowley, S. W. H., and E. J. Bunce (2001), Origin of the main auroral oval in Jupiter's coupled magnetosphere-ionosphere system, Planet. Space Sci., 49, 1067-1088, doi:10.1016/S0032-0633(00)00167-7.

Cowley, S. W. H., and E. J. Bunce (2003), Modulation of Jupiter's main auroral oval emissions by solar wind induced expansions and compressions of the magnetosphere, Planet. Space Sci., 51, 57-79, doi:10.1016/ S0032-0633(02)00118-6.

Cowley, S. W. H., E. J. Bunce, and J. D. Nichols (2003a), Origins of Jupiter's main auroral oval emissions, J. Geophys. Res., 108(A4), 8002, doi:10.1029/2002JA009329.

Cowley, S. W. H., E. J. Bunce, T. S. Stallard, and S. Miller (2003b), Jupiter's polar ionospheric flows: Theoretical interpretation, Geophys. Res. Lett., 30(5), 1220, doi:10.1029/2002GL016030.

Cowley, S. W. H., et al. (2005), A simple axisymmetric model of magnetosphere-ionosphere coupling currents in Jupiter's polar ionosphere, J. Geophys. Res., 110, A11209, doi:10.1029/2005JA011237.

Cravens, T. E. (1987), Vibrationally excited molecular hydrogen in the upper atmosphere of Jupiter, J. Geophys. Res., 92, 11,083-11,100, doi:10.1029/JA092iA10p11083.

Cravens, T. E., J. H. Waite, T. I. Gombosi, N. Lugaz, G. R. Gladstone, B. H. Mauk, and R. J. MacDowall (2003), Implications of Jovian X-ray emission for magnetosphere-ionosphere coupling, J. Geophys. Res., 108(A12), 1465, doi:10.1029/2003JA010050

Dickinson, R. E., E. C. Ridley, and R. G. Roble (1975), Meridional circulation in the thermosphere Part I. Equinox conditions, J. Atmos. Sci., 32, 1737-1754, doi:10.1175/1520-0469(1975)032<1737:MCITTI > 2.0.CO;2.

Dickinson, R. E., E. C. Ridley, and R. G. Roble (1984), Thermospheric general circulation model with coupled dynamics and composition, J. Atmos. Sci., 41, 205-219, doi:10.1175/1520-0469(1984)041<0205: TGCWCD $>2.0 . \mathrm{CO} ; 2$.

Dols, V., J.-C. Gérard, J. T. Clarke, J. Gustin, and D. Grodent (2000), Diagnostics of the Jovian aurora deduced from ultraviolet spectroscopy: Model and HST/GHRS observations, Icarus, 147, 251-266, doi:10.1006/icar.2000.6415.

Drossart, P., et al. (1989), Detection of $\mathrm{H}_{3}^{+}$on Jupiter, Nature, 340, 539-541, doi:10.1038/340539a0.

Drossart, P., B. Bezard, S. K. Atreya, J. Bishop, J. H. Waite Jr., and D. Boice (1993), Thermal profiles in the auroral regions of Jupiter, J. Geophys. Res., 98, 18,803-18,811, doi:10.1029/93JE01801.

Elsner, R. F., et al. (2005), Simultaneous Chandra X ray, Hubble Space Telescope ultraviolet, and Ulysses radio observations of Jupiter's aurora, J. Geophys. Res., 110, A01207, doi:10.1029/2004JA010717.

Eviatar, A., and A. D. Barbosa (1984), Jovian magnetospheric neutral wind and auroral precipitation flux, J. Geophys. Res., 89, 7393-7398, doi:10.1029/JA089iA09p07393.

Festou, M., S. K. Atreya, T. M. Donahue, B. R. Sandel, D. E. Shemansky, and A. L. Broadfoot (1981), Composition and thermal profile of the Jovian upper atmosphere determined by the Voyager stellar occultation experiments, J. Geophys. Res., 86, 5715-5725, doi:10.1029/ JA086iA07p05715.

Flasar, F. M., et al. (2004), An intense stratospheric jet on Jupiter, Nature, 427, 132-135, doi:10.1038/nature02142.

Frank, L. A., and W. R. Paterson (2001), Survey of thermal ions in the Io plasma torus with the Galileo spacecraft, J. Geophys. Res., 106, 6131-6150, doi:10.1029/2000JA000159.

Gérard, J.-C., D. Grodent, R. Prangé, J. H. Waite, G. R. Gladstone, V. Dols, F. Paresce, A. Stors, L. Ben Jaffel, and K. A. Franke (1994), A remarkable auroral event on Jupiter observed in the ultraviolet with the Hubble Space Telescope, Science, 266, 1675-1678, doi:10.1126/science. 266.5191 .1675 .

Gérard, J.-C., J. Gustin, D. Grodent, J. T. Clark, P. Delamere, and J. T. Clarke (2002), The excitation of the FUV Io tail on Jupiter: Characterization of the electron precipitation, J. Geophys. Res., 107(A11), 1394, doi:10.1029/ 2002JA009410.

Gérard, J.-C., J. Gustin, D. Grodent, and J. T. Clark (2003), Spectral observations of transient features in the FUV Jovian polar aurora, J. Geophys. Res., 108(A8), 1319, doi:10.1029/2003JA009901.

Gladstone, G. R., and T. E. Skinner (1988), Spectral analysis of Jovian auroral emissions, in Time-Variable Phenomena in the Jovian System, NASA Spec. Publ., vol. SP-494, edited by M. J. S. Belton, R. A. West, and J. Rahe, pp. 221-228, NASA, Washington, D. C.

Gladstone, G. R., M. Allen, and Y. L. Yung (1996), Hydrocarbon photochemistry in the upper atmosphere of Jupiter, Icarus, 119, 1-52, doi:10.1006/icar.1996.0001

Gladstone, G. R., J. H. Waite Jr., and J.-C. Gérard (1998), Jovian auroral Lya self-reversals: A window on Jupiter's auroral electrojet?, Bull. Am. Astron. Soc., 30, 1078

Gladstone, G. R., et al. (2002), A pulsating auroral X-ray hot spot on Jupiter, Nature, 415, 1000-1003, doi:10.1038/4151000a.

Grodent, D., J. H. Waite Jr., and J.-C. Gérard (2001), A self-consistent model of the Jovian auroral thermal structure, J. Geophys. Res., 106, 12,933-12,952, doi:10.1029/2000JA900129.

Grodent, D., J. T. Clarke, J. Kim, J. H. Waite, and S. W. H. Cowley (2003), Jupiter's main auroral oval observed with HST-STIS, J. Geophys. Res., 108(A11), 1389, doi:10.1029/2003JA009921.

Grodent, D., J.-C. Gérard, A. Radioti, B. Bonfond, and A. Saglam (2008), Jupiter's changing auroral location, J. Geophys. Res., 113, A01206, doi:10.1029/2007JA012601.

Gustin, J., D. Grodent, J.-C. Gérard, and J. T. Clarke (2002), Spatially resolved far ultraviolet spectroscopy of the Jovian aurora, Icarus, 157, 91-103, doi:10.1006/icar.2001.6784.

Gustin, J., et al. (2004a), Jovian auroral spectroscopy with FUSE: Analysis of self-absorption and implications for electron precipitation, Icarus, 171, 336-355, doi:10.1016/j.icarus.2004.06.005.

Gustin, J., et al. (2004b), Energy-flux relationship in the FUV Jovian aurora deduced from HST-STIS spectral observations, J. Geophys. Res., 109, A10205, doi:10.1029/2003JA010365.

Gustin, J., et al. (2006), Characteristics of Jovian morning bright FUV aurora from Hubble Space Telescope/Space Telescope Imaging Spectro- 
graph imaging and spectral observations, J. Geophys. Res., 111, A09220, doi:10.1029/2006JA011730.

Hickey, M. P., R. L. Walterscheid, and G. Schubert (2000), Gravity wave heating and cooling in Jupiter's thermosphere, Icarus, 148, 266-281, doi:10.1006/icar.2000.6472

Hill, T. W. (2001), The Jovian auroral oval, J. Geophys. Res., 106 , 8101-8108, doi:10.1029/2000JA000302.

Huang, T. S., and T. W. Hill (1989), Corotation lag of the Jovian atmosphere, ionosphere, and magnetosphere, J. Geophys. Res., 94, 3761-3765, doi:10.1029/JA094iA04p03761

Kane, M., B. H. Mauk, E. P. Keath, and S. M. Krimigis (1995), Hot ions in Jupiter's magnetodisc: A model for Voyager 2 low-energy charged particle measurements, J. Geophys. Res., 100, 19,473-19,486, doi:10.1029/ 95JA00793

Kharchenko, V., A. Dalgarno, D. R. Schultz, and P. C. Stancil (2006), Ion emission spectra in the Jovian X-ray aurora, Geophys. Res. Lett., 33 L11105, doi:10.1029/2006GL026039.

Khurana, K. K. (2001), Influence of solar wind on Jupiter's magnetosphere deduced from currents in the equatorial plane, J. Geophys. Res., 106 25,999-26,016, doi:10.1029/2000JA000352.

Kim, S. J., P. Drossart, J. Caldwell, J.-P. Maillard, T. Herbst, and M. Shure (1991), Images of aurorae on Jupiter from $\mathrm{H}_{3}^{+}$emission at 4 microns, Nature, 353, 536-539, doi:10.1038/353536a0.

Kim, Y. H., J. L. Fox, and H. S. Porter (1992), Densities and vibrational distribution of $\mathrm{H}_{3}^{+}$in the Jovian auroral ionosphere, J. Geophys. Res., 97 6093-6101, doi:10.1029/92JE00454.

Kim, Y. H., J. L. Fox, and J. J. Caldwell (1997), Temperatures and altitudes of Jupiter's ultraviolet aurora inferred from GHRS observations with the Hubble Space Telescope, Icarus, 128, 189-201, doi:10.1006/ icar.1997.5699.

Knight, S. (1973), Parallel electric fields, Planet. Space Sci., 21, 741-750, doi:10.1016/0032-0633(73)90093-7.

Krupp, N., et al. (2001), Global flows of energetic ions in Jupiter's equatorial plane: First-order approximation, J. Geophys. Res., 106, 26,017-26,032 doi:10.1029/2000JA900138.

Lam, H. A., N. Achilleos, S. Miller, J. Tennyson, L. M. Trafton, T. R. Geballe, and G. E. Ballester (1997), A baseline spectroscopic study of the infrared auroras of Jupiter, Icarus, 127, 379-393, doi:10.1006/ icar.1997.5698.

Liu, W., and A. Dalgarno (1996), The ultraviolet spectrum of the Jovian dayglow, Astrophys. J., 462, 502-518, doi:10.1086/177168.

Livengood, T. A., D. F. Strobel, and H. W. Moos (1990), Long-term study of longitudinal dependence in primary particle precipitation in the north Jovian aurora, J. Geophys. Res., 95, 10,375-10,388, doi:10.1029/ JA095iA07p10375.

Maillard, J. P., E. Lellouch, J. H. Waite Jr., B. Bézard, P. Drossart, J. Y. Mandin, and V. Dana (1999), Search for ionospheric winds in Jupiter from the $\mathrm{H}_{3}^{+}$emissions, Bull. Am. Astron. Soc., 31, 1186.

Majeed, T., and J. C. McConnell (1991), The upper ionosphere of Jupiter and Saturn, Planet. Space Sci., 39, 1715-1732, doi:10.1016/0032-0633 (91)90031-5

Majeed, T., J. C. McConnell, and G. R. Gladstone (1999), A model analysis of Galileo electron densities on Jupiter, Geophys. Res. Lett., 26, 2335-2338, doi:10.1029/1999GL900530.

Majeed, T., J. H. Waite, S. W. Bougher, R. V. Yelle, G. R. Gladstone, J. C. McConnell, and A. Bhardwaj (2004), The ionospheres-thermospheres of the giant planets, Adv. Space Res., 33, 197-211, doi:10.1016/ j.asr.2003.05.009.

Majeed, T., J. H. Waite, S. W. Bougher, and G. R. Gladstone (2005), Processes of equatorial thermal structure at Jupiter: An analysis of the Galileo temperature profile with a three-dimensional model, J. Geophys. Res., 110, E12007, doi:10.1029/2004JE002351.

Matcheva, K. I., and D. F. Strobel (1999), Heating of Jupiter's thermosphere by dissipation of gravity waves due to molecular viscosity and heat conduction, Icarus, 140, 328-340, doi:10.1006/icar.1999.6151.

Matsushita, S., J. D. Tarpley, and W. H. Campbell (1973), IMF sector structure effects on the quiet geomagnetic field, Radio Sci., 8, 963-972, doi:10.1029/RS008i011p00963.

Mauk, B. H., B. J. Anderson, and R. M. Thorne (2002), Magnetosphereionosphere coupling at Earth, Jupiter, and beyond, in Atmospheres in the Solar System: Comparative Aeronomy, Geophys. Monogr. Ser., vol. 130 edited by M. Mendillo, A. F. Nagy, and J. H. Waite, pp. 115-139, AGU, Washington, D. C

Maurellis, A. N., and T. E. Cravens (2001), Ionospheric effects of comet Shoemaker-Levy 9 impacts with Jupiter, Icarus, 154, 350-371, doi:10.1006/icar.2001.6709.

Melin, H., S. Miller, T. Stallard, and D. Grodent (2006), Estimated energy balance in the Jovian upper atmosphere during an auroral heating event, Icarus, 181, 256-265, doi:10.1016/j.icarus.2005.11.004.
Miller, S., H. A. Lam, and J. Tennyson (1994), What astronomy has learned from observations of $\mathrm{H}_{3}^{+}$, Can. J. Phys., 72, 760-771.

Miller, S., N. Achilleos, G. E. Ballester, H. A. Lam, J. Tennyson, T. R. Geballe, and L. M. Trafton (1997), Mid-to-low latitude $\mathrm{H}_{3}^{+}$emission from Jupiter, Icarus, 130, 57-67, doi:10.1006/icar.1997.5813.

Millward, G. H., S. Miller, T. Stallard, A. D. Aylward, and N. Achilleos (2002), On the dynamics of the Jovian ionosphere and thermosphere. Part III. The modelling of auroral conductivity, Icarus, 160, 95-107, doi:10.1006/icar.2002.6951

Millward, G. H., S. Miller, T. Stallard, N. Achilleos, and A. D. Aylward (2005), On the dynamics of the Jovian ionosphere and thermosphere. Part IV. Ion-neutral coupling, Icarus, 173, 200-211, doi:10.1016/ j.icarus.2004.07.027

Nichols, J. D., and S. W. H. Cowley (2004), Magnetosphere-ionosphere coupling currents in Jupiter's middle magnetosphere: Effect of precipitation-induced enhancement of the ionospheric Pedersen conductivity, Ann. Geophys., 22, 1799-1827.

Niemann, H. B., et al. (1996), The Galileo Probe Mass Spectrometer: Composition of Jupiter's atmosphere, Science, 272, 846-849, doi:10.1126/science.272.5263.846.

Orton, G. S., et al. (1991), Thermal maps of Jupiter: Spatial organization and time dependence of stratospheric temperatures, 1980-1990, Science, 252, 537-542, doi:10.1126/science.252.5005.537.

Pallier, L., and R. Prangé (2001), More about the structure of the high latitude Jovian aurorae, Planet. Space Sci., 49, 1159-1173, doi:10.1016/S0032-0633(01)00023-X.

Prangé, R., D. Rego, L. Pallier, L. Ben Jaffel, C. Emerich, J. T. Clarke, G. E. Ballester, and J. M. Ajello (1997), Detection of self-reversed Lyman $\alpha$ lines from the Jovian aurorae with the Hubble Space Telescope, Astrophys. J., 484, L169-L173, doi:10.1086/310786.

Prangé, R., D. Rego, L. Pallier, J. E. P. Connerney, P. Zarka, and J. Queinnec, (1998), Detailed study of FUV Jovian auroral features with the postCOSTAR HST faint object camera, J. Geophys. Res., 103, 20,195-20,216, doi:10.1029/98JE01128.

Radioti, A., J.-C. Gérard, D. Grodent, B. Bonfond, N. Krupp, and J. Woch (2008), Discontinuity in Jupiter's main auroral oval, J. Geophys. Res., 113, A01215, doi:10.1029/2007JA012610.

Raynaud, E., et al. (2003), The 10 October 1999 HIP9369 occultation by the northern polar region of Jupiter: Ingress and egress lightcurves analysis, Icarus, 162, 344-361, doi:10.1016/S0019-1035(03)00005-8.

Raynaud, E., K. Matcheva, P. Drossart, F. Roques, and B. Sicardy (2004a), A re-analysis of the 1971 Beta Scorpii occultation by Jupiter: Study of temperature fluctuations and detection of wave activity, Icarus, 168 324-335, doi:10.1016/j.icarus. 2003.10.021.

Raynaud, E., et al. (2004b), Spectro-imaging observations of Jupiter's $2-\mu \mathrm{m}$ auroral emission. Part $\mathrm{I} . \mathrm{H}_{3}^{+}$distribution and temperature, Icarus, 171, 133-152, doi:10.1016/j.icarus.2004.04.020.

Rego, D., N. Achilleos, T. Stallard, S. Miller, R. Prangé, M. Dougherty, and R. D. Joseph (1999), Supersonic winds in Jupiter's aurorae, Nature, 399, 121 - 124, doi:10.1038/20121.

Richmond, A. D., E. C. Ridley, and R. G. Roble (1992), A thermosphere/ ionosphere general circulation model with coupled electrodynamics, Geophys. Res. Lett., 19, 601-604, doi:10.1029/92GL00401.

Roble, R. G., and E. C. Ridley (1987), An auroral model for the NCAR thermospheric general circulation model (TGCM), Ann. Geophys., 5A, $369-382$.

Roble, R. G., and E. C. Ridley (1994), A thermosphere-ionospheremagnetosphere electrodynamics circulation model (TIME-GCM): Equinox solar minimum simulations (30-500 km), Geophys. Res. Lett., 21, 417-420, doi:10.1029/93GL03391.

Roble, R. G., E. C. Ridley, A. D. Richmond, and R. E. Dickinson (1988), A coupled thermospheric-ionospheric general circulation model, Geophys. Res. Lett., 15, 1325-1328, doi:10.1029/GL015i012p01325.

Satoh, T., and J. E. P. Connerney (1999), Jupiter's $\mathrm{H}_{3}^{+}$emissions viewed in corrected Jovimagnetic coordinates, Icarus, 141, 236-252, doi:10.1006/ icar.1999.6173.

Satoh, T., J. E. P. Connerney, and R. L. Baron (1996), Emission source model of Jupiter's $\mathrm{H}_{3}^{+}$aurorae: A generalized inverse analysis of images, Icarus, 122, 1-23, doi:10.1006/icar.1996.0106.

Seiff, A., D. B. Kirk, T. C. D. Knight, R. E. Young, J. D. Mihalov, L. A.Young, F. S. Milos, G. Schubert, R. C. Blanchard, and D. Atkinson (1998), Thermal structure of Jupiter's atmosphere near the edge of $5-\mu \mathrm{m}$ hot spot in the north equatorial belt, J. Geophys. Res., 103, 22,857-22,890, doi:10.1029/98JE01766.

Smith, C. G. A., A. D. Aylward, G. H. Millward, S. Smith, and L. E. Moore (2007), An unexpected cooling effect in Saturn's upper atmosphere, Nature, 445, 399-401, doi:10.1038/nature05518.

Sommeria, J., L. Ben-Jaffel, and R. Prange (1995), On the existence of supersonic jets in the upper atmosphere of Jupiter, Icarus, 118, 2-24, doi:10.1006/icar. 1995.1174 
Stallard, T., S. Miller, G. Millward, and R. D. Joseph (2001), On the dynamics of the Jovian ionosphere and thermosphere. Part I. The measurement of ion winds, Icarus, 154, 475-491, doi:10.1006/icar.2001.6681.

Stallard, T., S. Miller, G. Millward, and R. D. Joseph (2002), On the dynamics of the Jovian ionosphere. Part II. The measurements of $\mathrm{H}_{3}^{+}$ vibrational temperature, column density and total emission, Icarus, 156, 498-514, doi:10.1006/icar.2001.6793.

Strobel, D. F., and G. R. Smith (1973), On the temperature of the Jovian thermosphere, J. Atmos. Sci., 30, 718-725, doi:10.1175/1520-0469 (1973) $030<0718:$ OTTOTJ $>2.0 . C O ; 2$.

Trafton, L. M., J.-C. Gérard, G. Munhoven, and J. H. Waite (1994), Highresolution spectra of Jupiter's northern auroral ultraviolet emission with the Hubble Space Telescope, Astrophys. J., 421, 816-827, doi:10.1086/ 173694.

Trafton, L. M., V. Dols, J.-C. Gérard, J. H. Waite, G. R. Gladstone, and G. Munhoven (1998), HST spectra of the Jovian ultraviolet aurora: Search for heavy ion precipitation, Astrophys. J., 507, 955-967, doi:10.1086/306338

Vasyliunas, V. M. (2001), Electric field and plasma flow: What drives what?, Geophys. Res. Lett., 28, 2177-2180, doi:10.1029/2001GL013014.

Veverka, J., L. H. Wasserman, J. Elliot, and C. Sagan (1974), The occultation of $\beta$ Scorpii by Jupiter. Part I. The structure of the Jovian upper atmosphere, Astron. J., 79, 73-84, doi:10.1086/111534.

Waite, J. H., Jr., and D. Lummerzheim (2002), Comparison of auroral processes: Earth and Jupiter, in Atmospheres in the Solar System: Comparative Aeronomy, Geophys. Monogr. Ser., vol. 130, edited by M. Mendillo, A. F. Nagy, and J. H. Waite, pp. 115-139, AGU, Washington, D. C.

Waite, J. H., Jr., T. E. Cravens, J. U. Kozyra, A. F. Nagy, S. K. Atreya, and R. H. Chen (1983), Electron precipitation and related aeronomy of the
Jovian thermosphere and ionosphere, J. Geophys. Res., 88, 6143-6163, doi:10.1029/JA088iA08p06143.

Waite, J. H., Jr., et al. (2000), Multispectral observations of Jupiter's aurora, Adv. Space Res., 26, 1453-1475, doi:10.1016/S0273-1177(00)00089-2.

Waite, J. H., Jr., et al. (2001), An auroral flare at Jupiter, Nature, 410, 787-789, doi:10.1038/35071018.

Yelle, R. V., C. A. Griffith, and L. A. Young (2001), Structure of the Jovian stratosphere at the Galileo probe entry site, Icarus, 152, 331-346, doi:10.1006/icar.2001.6640.

Young, L. A., R. V. Yelle, R. Young, A. Sieff, and D. B. Kirk (1997) Gravity waves in Jupiter's thermosphere, Science, 276, 108-111, doi:10.1126/science.276.5309.108.

Young, L. A., R. V. Yelle, R. Young, A. Sieff, and D. B. Kirk (2005), Gravity waves in Jupiter's stratosphere, as measured by the Galileo ASI experiment, Icarus, 173, 185-199, doi:10.1016/j.icarus.2004.07.020

Yung, Y. L., G. R. Gladstone, K. M. Chang, J. M. Ajello, and S. K. Srivastava (1982), $\mathrm{H}_{2}$ fluorescence spectrum from 1200 to $1700 \AA$ by electron impact: Laboratory study and application to Jovian aurora, Astrophys. J., 254, L65-L69, doi:10.1086/183757.

S. W. Bougher and T. Majeed, Department of Atmospheric, Oceanic and Space Sciences, University of Michigan, 2455 Hayward Street, Ann Arbor MI 48109-2143, USA. (tariqm@umich.edu)

G. R. Gladstone and J. H. Waite, Southwest Research Institute, 6220 Culebra Road, P.O. Box Drawer 28610, San Antonio, TX 78228-0510, USA. 